\title{
Dynamical core model intercomparison project: Tracer transport test cases
}

\author{
James Kent, ${ }^{\text {a }}$ Paul A. Ullrich ${ }^{\mathrm{a}, \mathrm{b}}$ and Christiane Jablonowski ${ }^{\mathrm{a}}$ \\ ${ }^{a}$ Department of Atmospheric, Oceanic and Space Sciences, University of Michigan, Ann Arbor, MI, USA \\ ${ }^{\mathrm{b}}$ Department of Land, Air and Water Resources, University of California, Davis, CA, USA
}

${ }^{*}$ Correspondence to: J. Kent, Department of Atmospheric, Oceanic and Space Sciences, University of Michigan, 2455 Hayward St, Ann Arbor, MI 48109-2143, USA. E-mail: jdkent@umich.edu

\begin{abstract}
Three-dimensional advection tests are required to assess the ability of transport schemes of dynamical cores to model tracer transport on the sphere accurately. A set of three tracertransport test cases for three-dimensional flow is presented. The tests focus on the physical and numerical issues that are relevant to three-dimensional tracer transport: positivity preservation, inter-tracer correlations, horizontal-vertical coupling, order of accuracy and choice of vertical coordinate. The first test is a three-dimensional deformational flow. The second test is a Hadley-like global circulation. The final test is a solid-body rotation test in the presence of rapidly varying orography. A variety of assessment metrics, such as error norms, convergence rates and mixing diagnostics, are used. The tests are designed for easy implementation within existing and developing dynamical cores and have been a cornerstone of the 2012 Dynamical Core Model Intercomparison Project (DCMIP). Example results are shown using the transport schemes in two dynamical cores: the Community Atmosphere Model finite-volume dynamical core (CAM-FV) and the cubed-sphere finite-volume MCore dynamical core.
\end{abstract}

Key Words: advection; three-dimensional flow; spherical geometry; finite-volume methods

Received 11 February 2013; Revised 9 May 2013; Accepted 2 June 2013; Published online in Wiley Online Library 19 July 2013

\section{Introduction}

Significant research has gone into the development of state-ofthe-art transport schemes on the sphere, for use in weather and climate models. Tracer transport is performed by the dynamical core, the fluid dynamics component of a general circulation model (GCM), and is very important in atmospheric models. The transport scheme is used to advect the many tracer species that are used in climate models and climate prediction studies (Lamarque et al., 2008). It is strongly linked to the chemistry module, with errors due to the numerical transport scheme having a large impact on errors in chemistry models and certain physical parametrizations (Prather et al., 2008; Ovtchinnikov and Easter, 2009; Plumb et al., 2000). There are many different numerical methods for tracer transport (e.g. finite-volume (Lin and Rood, 1996), discontinuous Galerkin (Nair et al., 2005) and semi-Lagrangian (Zerroukat et al., 2002); see Rood (1987) for a review), used on different spherical grids (Staniforth and Thuburn, 2012), that are employed by dynamical cores. Consequently, it is essential to be able to assess and evaluate these numerical methods.

To assess the characteristics of the numerical transport scheme, testing is performed on idealized test cases. This requires a prescribed velocity component and preferably a known solution. Although there are many two-dimensional horizontal tracer test cases on the sphere, including simple solid-body rotation tests (Williamson et al., 1992), static and moving vortices (Nair and Machenhauer, 2002; Nair and Jablonowski, 2008) and deformational flows (Nair and Lauritzen, 2010; Kent et al., 2012b), very few fully three-dimensional tracer-transport tests have been offered. Examples include solid-body rotation with a sinusoidal vertical velocity (Hubbard, 2002) and the three-dimensional advection tests of Zubov et al. (1999). Other test scenarios assess tracers in either idealized adiabatic flows (Whitehead et al., 2013; Kent et al., 2012a) or a full model simulation (Rasch et al., 2006). This article aims at suggesting a set of three complex threedimensional transport test cases with prescribed velocities on the sphere. These test cases are specifically designed to test the properties relevant to the design of physically realistic transport schemes, namely positivity and monotonicity, preservation of existing tracer correlations (see Thuburn and McIntyre, 1997), horizontal-vertical coupling and the transport of tracers over orography.

The advection process can be represented in many forms. For a given tracer mixing ratio $q$, the advection equation can be expressed as

$$
\begin{aligned}
\frac{\mathrm{D} q}{\mathrm{D} t} & =0, \\
\frac{\partial q}{\partial t}+\mathbf{v} \cdot \nabla q & =0,
\end{aligned}
$$


in a Lagrangian and Eulerian framework, respectively. Here, $\mathbf{v}$ symbolizes the three-dimensional wind vector, $\nabla$ is the threedimensional gradient operator and $\mathrm{D} / \mathrm{D} t$ stands for the material time derivative.

If an advection scheme utilizes the conservation form

$$
\frac{\partial}{\partial t}(\rho q)+\nabla \cdot(\mathbf{v} \rho q)=0
$$

the air density $\rho$ needs to be present so that the equation represents a tracer mass density. The mass continuity equation that is solved for $\rho$ is given as

$$
\frac{\partial \rho}{\partial t}+\nabla \cdot(\mathbf{v} \rho)=0
$$

However, the tests in this article are designed so that $(\mathbf{v} \rho)$ is a divergence-free field, i.e. the continuity equation is analytically satisfied even without the constraint of constant density, with

$$
\frac{\partial \rho}{\partial t}=0
$$

This article describes the initial state, velocity fields and diagnostics of three new three-dimensional tracer-transport test cases. These tests are designed to return the tracers to their initial position at the end of the simulation, thus providing a final reference solution. This analytic reference solution is a key advantage of this test suite and allows the straightforward calculation of error norms. These tracer-transport tests have been developed for the 2012 Dynamical Core Model Intercomparison Project (DCMIP).* We provide example results from the transport schemes of two dynamical cores that participated in DCMIP: the Community Atmosphere Model finite-volume dynamical core (CAM-FV: Lin, 2004) and the cubed-sphere finite-volume MCore dynamical core (Ullrich and Jablonowski, 2012a). Section 2 provides an overview of the general set-up of the test cases and a description of the two dynamical cores. The three test-case descriptions and example results are given in sections 3, 4 and 5. We also provide FORTRAN initialization routines in the Supporting Information so as to ease practical implementation.

\section{Overview of the DCMIP tracer-transport test cases set-up}

This section describes the general set-up for the three-dimensional passive advection tests. Each test makes use of prescribed wind fields. We apply time reversal (overlaid with a solid-body rotation) to return the tracer to its original position in two of the suggested tests, while the third test utilizes a two-dimensional solid-body rotation and returns the tracer to its initial position after one revolution around the sphere. This ensures that an analytical solution is known at the end of the simulation for each test.

The tracer-transport tests are designed to be implemented directly into the dynamical cores of GCMs. The first test, 1-1, is a three-dimensional flow that extends the two-dimensional deformation test proposed by Nair and Lauritzen (2010). The second test, 1-2, focuses on the horizontal-vertical coupling of the advection scheme, which is an important issue in atmospheric modelling, as many dynamical cores are horizontally-vertically dimension-split. The final test, $1-3$, uses three-dimensional flow in the presence of orography and is used to test models that utilize terrain-following vertical coordinates. The numbering convention of the test cases $(1-1,1-2$ and $1-3)$ is based on the numbering of the tests used at DCMIP in 2012. As mentioned before, the tests make use of prescribed three-dimensional

\footnotetext{
* More information about DCMIP and its associated two-week workshop in the summer of 2012 can be found at http://earthsystemcog.org/projects/dcmip$2012 /$.
}

Table 1. A list of physical constants used herein.

\begin{tabular}{lll}
\hline Constant & Description & Value \\
\hline$a$ & Radius of the Earth & $6.37122 \times 10^{6} \mathrm{~m}$ \\
$g$ & Gravity & $9.80616 \mathrm{~m} \mathrm{~s}^{-2}$ \\
$p_{0}$ & Reference pressure & $1000 \mathrm{hPa}$ \\
$c_{\mathrm{p}}$ & Specific heat capacity of dry air & $1004.5 \mathrm{~J} \mathrm{~kg}^{-1} \mathrm{~K}^{-1}$ \\
$R_{\mathrm{d}}$ & Gas constant for dry air & $287.0 \mathrm{~J} \mathrm{~kg}^{-1} \mathrm{~K}^{-1}$ \\
$\kappa$ & Ratio of $R_{\mathrm{d}}$ to $c_{\mathrm{p}}$ & $R_{\mathrm{d}} / c_{\mathrm{p}}=2 / 7$ \\
$z_{\text {top }}$ & Height position of the model top & $12000 \mathrm{~m}$ \\
$p_{\text {top }}$ & Pressure at the model top & $\approx 254.944 \mathrm{hPa}$ \\
$T_{0}$ & Isothermal atmospheric tempera- & $300 \mathrm{~K}$ \\
& ture & \\
\hline
\end{tabular}

velocities and an isothermal temperature field. Consequently, dynamic updates of the velocity, temperature and pressure fields need to be disabled and prescribed (analytic) updates of the time-dependent velocity fields need to be included into the model code for test $1-1$ and $1-2$. Test $1-3$ utilizes time-independent velocities that can be provided via the initial data set. A list of physical constants used throughout this article is given in Table 1. Constants specific to each test case are similarly tabulated at the beginning of each section.

The analytic initial conditions are described in terms of latitude $\varphi$, longitude $\lambda$ and either height $z$ or pressure $p$. The pressure field is prescribed and needs to remain constant for the duration of the simulation, except if floating Lagrangian pressure-based coordinates are used in the vertical direction (Lin, 2004), as explained in Appendix A. Advection schemes in the latter framework may require prescribed variations of the pressure thicknesses $\Delta p$ between two model interface levels to account for deforming layers. Such a deformation for floating Lagrangian coordinates will only be valid for one time step before a vertical remapping algorithm restores the initial pressure values at the model levels.

The pressure field is given by

$$
p(\lambda, \varphi, z, t)=p_{0} \exp \left(\frac{-g z}{R_{\mathrm{d}} T_{0}}\right),
$$

where $T_{0} \equiv 300 \mathrm{~K}$ is the isothermal atmospheric temperature that yields $T(\lambda, \varphi, z, t)=T_{0}$ for all three test variants, $R_{\mathrm{d}}$ is the gas constant for dry air and $g$ symbolizes gravity. The reference pressure at $z=0 \mathrm{~m}$ is set to $p_{0}=1000 \mathrm{hPa}$. The surface pressure $p_{\mathrm{s}}$, which may be needed for initializing hydrostatic models, can be computed when evaluating (6) at the surface elevation $z_{\mathrm{s}}$, which is specified later. Note that (6) can also be expressed as

$$
z(\lambda, \varphi, p)=H \ln \left(\frac{p_{0}}{p}\right)
$$

which utilizes the scale height

$$
H \equiv \frac{R_{\mathrm{d}} T_{0}}{g} .
$$

Equation (7) transforms the pressure into the height $z$ in an isothermal atmosphere.

For models that solve the advective form of the transport equation (2), the density does not require consideration, but for models that solve the conservative form (3) the air density is required. In order to avoid solving a second transport equation for $\rho$ and to simplify the test set-up, the stratified density is defined as

$$
\begin{aligned}
& \rho(\lambda, \varphi, p)=\frac{p}{R_{\mathrm{d}} T_{0}}, \\
& \rho(\lambda, \varphi, z)=\frac{p_{0}}{R_{\mathrm{d}} T_{0}} \exp \left(\frac{-z}{H}\right),
\end{aligned}
$$


for models with pressure-based or height-based coordinates, respectively. For all tests, the density should be held constant $(\partial \rho / \partial t=0)$ for the duration of the experiment. The velocity field for each test is chosen to satisfy the non-divergent condition exactly, i.e. in vertical pressure coordinates it yields

$$
\frac{1}{a \cos \varphi}\left[\frac{\partial u}{\partial \lambda}+\frac{\partial}{\partial \varphi}(v \cos \varphi)\right]+\frac{\partial \omega}{\partial p}=0,
$$

where $u$ is the zonal velocity, $v$ the meridional velocity, $\omega$ the vertical pressure velocity and $a$ is the radius of the Earth. In height coordinates, the relationship is given by

$$
\frac{1}{a \cos \varphi}\left[\frac{\partial(\rho u)}{\partial \lambda}+\frac{\partial}{\partial \varphi}(\rho v \cos \varphi)\right]+\frac{\partial(\rho w)}{\partial z}=0,
$$

where $w$ is the vertical velocity. This will ensure that models with pressure-based and height-based vertical coordinates will resemble each other, since isothermal conditions are used to determine the placement of the initial pressure levels. For models that utilize the conservation form of the advection equation, it may be beneficial to run each of the tests with the optional tracer field

$$
q_{0}(\lambda, \varphi, z)=1
$$

which tests how well the model is able to satisfy the threedimensional continuity equations (4) and (10).

Normalized error norms are used in all three sets of tests. They are defined by

$$
\begin{aligned}
\ell_{1}(q) & =\frac{I\left[\left|q-q_{\mathrm{T}}\right|\right]}{I\left[\left|q_{\mathrm{T}}\right|\right]}, \\
\ell_{2}(q) & =\sqrt{\frac{I\left[\left(q-q_{\mathrm{T}}\right)^{2}\right]}{I\left[q_{\mathrm{T}}^{2}\right]}}, \\
\ell_{\infty}(q) & =\frac{\max \left|q-q_{\mathrm{T}}\right|}{\max \left|q_{\mathrm{T}}\right|},
\end{aligned}
$$

where $q_{\mathrm{T}}$ is the tracer field at the initial time (due to the periodicity of the test cases, this is also the exact solution). Here $I$ denotes an approximation to the global integral, given by

$$
I[X]=\sum_{\text {all elements } j} X_{j} V_{j},
$$

where $V_{j}$ denotes the volume of element $j$.

\subsection{Brief description of the dynamical cores}

\subsubsection{CAM-FV}

The Community Atmosphere Model finite-volume dynamical core is an operational dynamical core in the National Center for Atmospheric Research's Community Earth System Model (Neale et al., 2010) and is described in detail in Lin (2004). The horizontal tracer transport component is based upon the fluxform semi-Lagrangian method as described in Lin and Rood (1996). A floating Lagrangian coordinate is used in the vertical, which is periodically remapped to a fixed grid. This means that CAM-FV solves the transport equation as given by (A1) in Appendix A. In the presence of orography, terrain-following hybrid coordinates are used (Simmons and Burridge, 1981). Variations of the PPM algorithm (Colella and Woodward, 1984)

\begin{tabular}{|c|c|c|}
\hline Constant & Value & Description \\
\hline$\tau$ & $1036800 \mathrm{~s}$ & Period of motion (here 12 days) \\
\hline$\omega_{0}$ & $23000 \pi / \tau$ & $\begin{array}{l}\text { Maximum of the vertical pressure } \\
\text { velocity in units of } \mathrm{Pa} \mathrm{s}^{-1}\end{array}$ \\
\hline$b$ & 0.2 & $\begin{array}{l}\text { Normalized pressure depth of the } \\
\text { divergent layer }\end{array}$ \\
\hline$\lambda_{\mathrm{cl}}$ & $5 \pi / 6$ & Initial longitude of first tracer \\
\hline$\lambda_{\mathrm{c} 2}$ & $7 \pi / 6$ & Initial longitude of second tracer \\
\hline$\varphi_{\mathrm{c}}$ & 0 & Initial latitude of tracers \\
\hline$z_{\mathrm{c}}$ & $5000 \mathrm{~m}$ & Initial altitude of tracers \\
\hline$R_{\mathrm{t}}$ & $a / 2$ & Horizontal half-width of tracers \\
\hline$Z_{\mathrm{t}}$ & $1000 \mathrm{~m}$ & Vertical half-width of tracers \\
\hline
\end{tabular}
are used both to calculate the numerical fluxes in the Lin-Rood scheme and in the vertical remapping. A filling algorithm is also present, to prevent any negative tracer values. CAM-FV makes use of the latitude-longitude grid.
Table 2. List of constants used for the three-dimensional deformational flow test case (Test 1-1)

\subsubsection{MCore}

MCore, described by Ullrich and Jablonowski (2012a), uses high-order upwind finite-volume methods (Ullrich et al., 2010; Ullrich and Jablonowski, 2012b) on the cubed-sphere grid (Rancic et al., 1996). A fourth-order three-dimensional discretization that captures the horizontal cross-terms is used. Note that this differs from the second-order vertical discretization described in Ullrich and Jablonowski (2012a). The finite-volume method provides implicit diffusion through a modified version of the low-speed Advection Upstream Splitting Method $\left(\mathrm{AUSM}^{+}\right)$up Riemann solver. A filter is used to ensure positivity (note that a monotonic filter is optional but not used in the tests in this document). Panel edges of the cubed-sphere grid are treated using a fourth-order remapping scheme. The vertical coordinate uses the Gal-Chen (Gal-Chen and Somerville, 1975) formulation. As MCore solves the flux form of equation (3) for tracer density, division by $\rho$ must take place to output the mixing ratio $q$. Unless stated otherwise, for MCore the tracer density is divided by the analytical density (10) (which remains constant with time), not the numerical density, which can be calculated using $q_{0}$ (which will not remain constant with time, due to numerical error). The solution from MCore is analyzed on the native cubed-sphere grid but interpolated to the same latitude-longitude grid as CAM-FV for visualization.

\section{Test 1-1: Three-dimensional deformational flow}

The three-dimensional deformational flow test is an extension of the two-dimensional approach of test case 4 by Nair and Lauritzen (2010), with an additional prescribed vertical wind velocity and corresponding horizontally divergent wind field. The test also provides a measure of the transport scheme's ability to maintain nonlinear tracer correlations, using the mixing diagnostics developed by Lauritzen and Thuburn (2012). These mixing diagnostics are a method for determining the nature of the numerical mixing errors introduced by an advection scheme. These errors are of particular importance in atmospheric chemistry modelling, since they represent important functional relationships between tracer species (Plumb and Ko, 1992; Thuburn and McIntyre, 1997). The list of constants used in test $1-1$ is given in Table 2 .

The test utilizes a translational longitude, defined by

$$
\lambda^{\prime}=\lambda-2 \pi t / \tau
$$

where $t$ denotes the elapsed time since the start of the simulation and $\tau$ denotes the period for the simulation to return to its initial state. The vertical pressure velocity is specified as

$$
\omega(\lambda, \varphi, p, t)=\omega_{0} \sin \lambda^{\prime} \cos \varphi \cos \left(\frac{2 \pi t}{\tau}\right) s(p),
$$



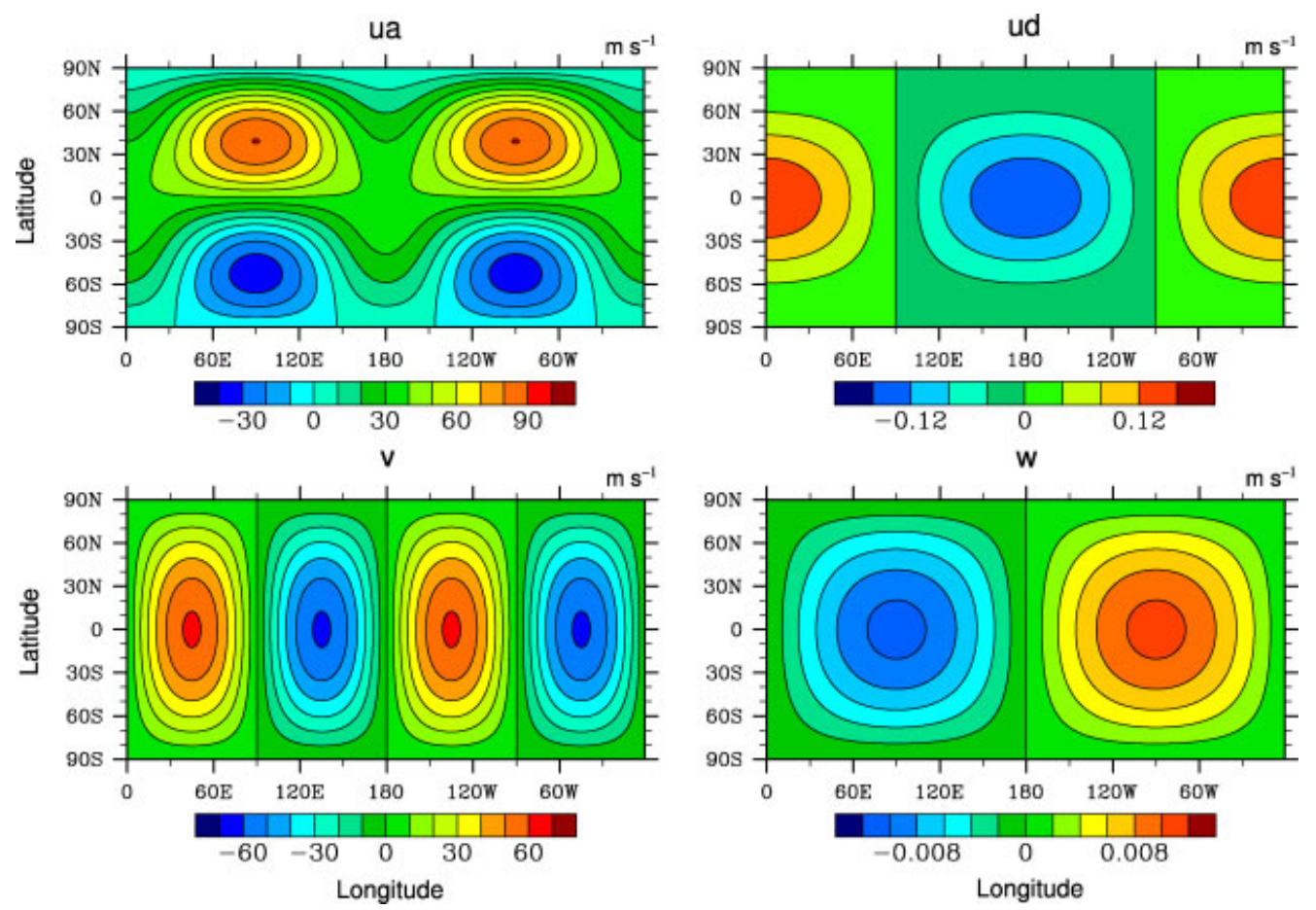

Figure 1. Test 1-1 initial conditions: latitude-longitude plots of velocities $u_{\mathrm{a}}, u_{\mathrm{d}}, v$ and $w$ at $4900 \mathrm{~m}$. This figure is available in colour online at wileyonlinelibrary.com/journal/qj

where

$$
\begin{aligned}
s(p)= & 1+\exp \left(\frac{p_{\text {top }}-p_{0}}{b p_{\text {top }}}\right)-\exp \left(\frac{p-p_{0}}{b p_{\text {top }}}\right) \\
& -\exp \left(\frac{p_{\text {top }}-p}{b p_{\text {top }}}\right)
\end{aligned}
$$

is a smooth tapering function that tapers the vertical velocity to zero at the top and bottom of the domain. Since pressure and height surfaces are aligned, the pressure position of the model top, $p_{\text {top }}$, is

$$
p_{\text {top }}=p\left(z_{\text {top }}\right)
$$

where pressure is determined by (6). In terms of the translational longitude, the horizontal zonal and meridional velocities $\mathbf{u}=$ $(u, v)$ are given as the sum of a horizontal deformational component $\mathbf{u}_{\mathrm{a}}=\left(u_{\mathrm{a}}, v_{\mathrm{a}}\right)$ and a horizontally divergent component $\mathbf{u}_{\mathrm{d}}=\left(u_{\mathrm{d}}, v_{\mathrm{d}}\right)$ :

$$
\mathbf{u}=\mathbf{u}_{\mathrm{a}}+\mathbf{u}_{\mathrm{d}}
$$

The deformational zonal and meridional wind components follow from Nair and Lauritzen (2010):

$$
\begin{aligned}
& u_{\mathrm{a}}(\lambda, \varphi, p, t)=\frac{10 a}{\tau} \sin ^{2}\left(\lambda^{\prime}\right) \sin (2 \varphi) \cos (\pi t / \tau)+\frac{2 \pi a}{\tau} \cos \varphi \\
& v_{\mathrm{a}}(\lambda, \varphi, p, t)=\frac{10 a}{\tau} \sin \left(2 \lambda^{\prime}\right) \cos (\varphi) \cos (\pi t / \tau)
\end{aligned}
$$

The two-dimensional divergent wind component is given by

$$
\begin{aligned}
u_{\mathrm{d}}(\lambda, \varphi, p, t)= & \frac{\omega_{0} a}{b p_{\text {top }}} \cos \left(\lambda^{\prime}\right) \cos ^{2}(\varphi) \cos \left(\frac{2 \pi t}{\tau}\right) \\
& \times\left[-\exp \left(\frac{p-p_{0}}{b p_{\text {top }}}\right)+\exp \left(\frac{p_{\text {top }}-p}{b p_{\text {top }}}\right)\right],
\end{aligned}
$$

$v_{\mathrm{d}}(\lambda, \varphi, p, t)=0$
The total velocity field is chosen to satisfy $\nabla \cdot(\mathbf{v} \rho)=0$ exactly. The surface is flat, with $z_{\mathrm{s}}=0 \mathrm{~m}$ or equivalently surface geopotential $\Phi_{\mathrm{s}}=0 \mathrm{~m}^{2} \mathrm{~s}^{-2}$. The surface pressure is constant with $p_{\mathrm{s}}(\lambda, \varphi)=p_{0}$. Therefore, the vertical velocity for models with vertical $\sigma$ (Phillips, 1957) or hybrid $\sigma$-pressure $(\eta)$ coordinates (Simmons and Burridge, 1981) is

$$
\dot{\eta}(\lambda, \varphi, \eta, t)=\dot{\sigma}(\lambda, \varphi, \sigma, t)=\frac{\omega}{p_{0}},
$$

where $\eta$ and $\sigma$ are given by $\eta=\sigma=p / p_{0}$. Note that this formulation assumes that the reference pressure for the hybrid $\eta$ coordinate is set to $1000 \mathrm{hPa}$. Since there are neither time variations nor horizontal variations of the pressure field, the vertical velocity in height coordinates takes the simple form

$$
w(\lambda, \varphi, z, t)=-\frac{\omega(\lambda, \varphi, p(z), t)}{g \rho(z)},
$$

with $\rho$ given by the density equation (10).

The initial velocities $u_{\mathrm{a}}, u_{\mathrm{d}}, v$ and $w$ are shown in Figure 1 at the $4900 \mathrm{~m}$ height level. Note that at this height $u_{\mathrm{d}}$ is two orders of magnitude smaller than $u_{\mathrm{a}}$, meaning that the horizontal velocities act almost identically to those in Nair and Lauritzen (2010).

Four tracer mixing ratios are specified for this test. The first tracer field represents two cosine bells, and is specified as

$$
q_{1}(\lambda, \varphi, z)=\frac{1}{2}\left[1+\cos \left(\pi d_{1}\right)\right]+\frac{1}{2}\left[1+\cos \left(\pi d_{2}\right)\right],
$$

where $d_{i}(i=1,2)$ denote the scaled distance functions,

$$
d_{i}(\lambda, \varphi, z)=\min \left[1,\left\{\left(\frac{r_{i}(\lambda, \varphi)}{R_{\mathrm{t}}}\right)^{2}+\left(\frac{z-z_{\mathrm{c}}}{Z_{\mathrm{t}}}\right)^{2}\right\}\right]
$$

and $r_{i}(\lambda, \varphi)(i=1,2)$ denotes the great circle distance,

$$
r_{i}(\lambda, \varphi)=a \arccos \left[\sin \varphi_{\mathrm{c}} \sin \varphi+\cos \varphi_{\mathrm{c}} \cos \varphi \cos \left(\lambda-\lambda_{\mathrm{ci}}\right)\right]
$$



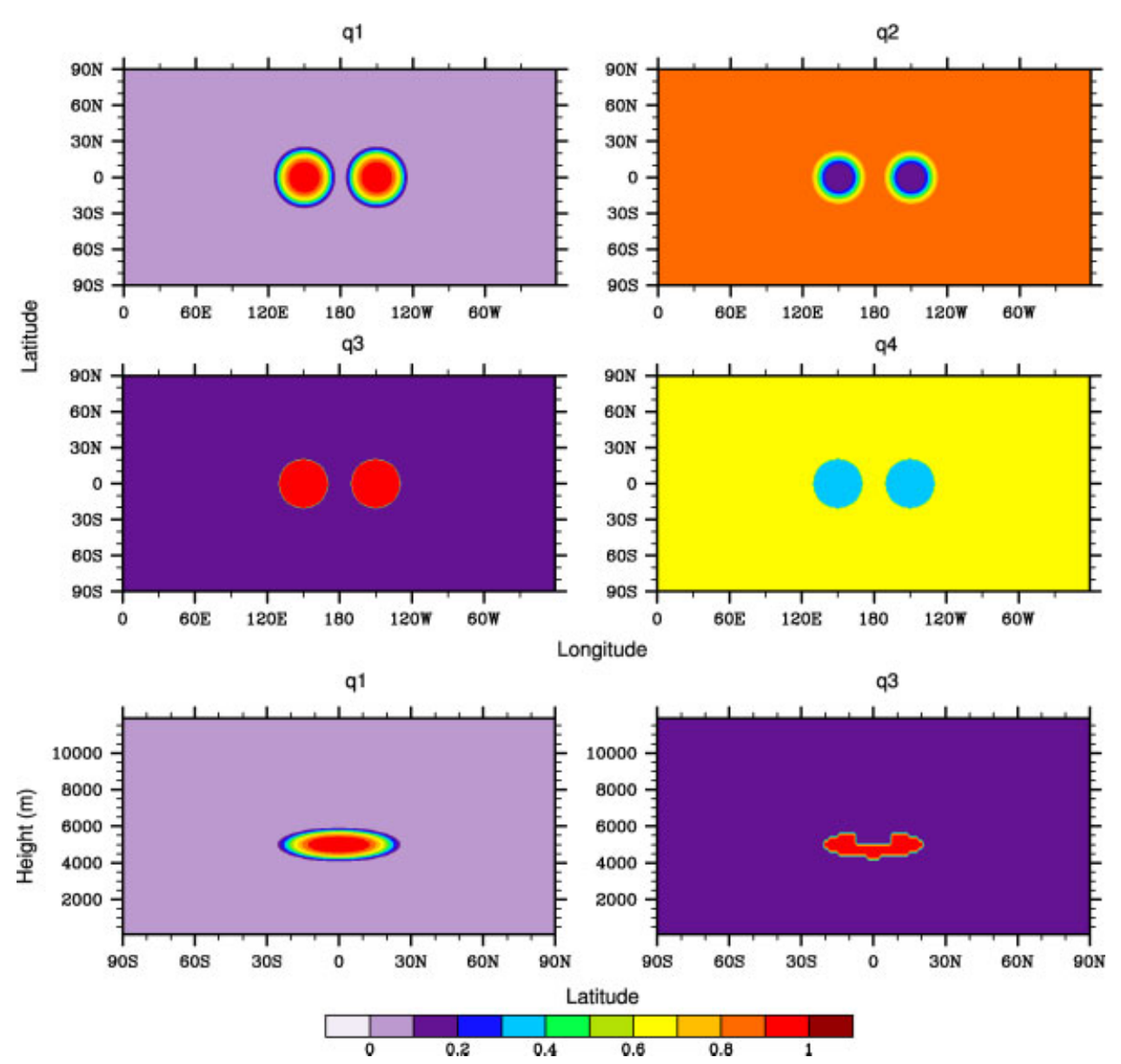

Figure 2. Test 1-1 initial conditions: latitude-longitude plots of tracers $q_{1}, q_{2}, q_{3}$ and $q_{4}$ at $4900 \mathrm{~m}$ and latitude-height cross-sections of $q_{1}$ and $q_{3}$ at longitude $\lambda=\lambda_{\mathrm{cl}}$. This figure is available in colour online at wileyonlinelibrary.com/journal/qj

The second tracer is chosen to assess the ability of the transport scheme to maintain a nonlinear correlation with the first tracer. By defining nonlinearly correlated tracer fields $\left(q_{1}, q_{2}\right)=[\chi, \psi(\chi)]$, one can determine how well the numerical scheme preserves these correlations over the duration of the simulation. The second tracer is thus initialized as

$$
q_{2}(\lambda, \varphi, z)=0.9-0.8 q_{1}(\lambda, \varphi, z)^{2} .
$$

The third tracer is used to assess the capability of a transport scheme to achieve monotonicity and is set up as two slotted ellipses:

$$
q_{3}(\lambda, \varphi, z)= \begin{cases}1 & \text { if } d_{1}<1 / 2 \\ 1 & \text { if } d_{2}<1 / 2 \\ 0.1 & \text { otherwise }\end{cases}
$$

with the additional condition

$$
q_{3}(\lambda, \varphi, z)=0.1 \text { if } z>z_{\mathrm{c}} \text { and } \varphi_{\mathrm{c}}-1 / 8<\varphi<\varphi_{\mathrm{c}}+1 / 8 \text {. }
$$

The final tracer is chosen to investigate whether the linear sum of multiple tracers can be maintained by the transport scheme (Lauritzen and Thuburn, 2012). It is set up so that, in combination with the other tracer fields with weight $(3 / 10)$, the sum is equal to 1 :

$$
q_{4}(\lambda, \varphi, z)=1-\frac{3}{10}\left[q_{1}(\lambda, \varphi, z)+q_{2}(\lambda, \varphi, z)+q_{3}(\lambda, \varphi, z)\right] .
$$

The top and middle plots of Figure 2 show the initial tracers $q_{1}, q_{2}, q_{3}$ and $q_{4}$ at a height level of $4900 \mathrm{~m}$. The bottom plots of Figure 2 show latitude-height cross-sections of $q_{1}$ and $q_{3}$ at longitude $\lambda=\lambda_{\mathrm{cl}}$. The plots are generated on $1^{\circ} \times 1^{\circ}$ resolution grid with 60 vertical levels.

\subsection{Grid spacings and diagnostics}

For purposes of model intercomparison, this test should be run at $1^{\circ} \times 1^{\circ}$ resolution $(\sim 110 \mathrm{~km}$ equatorial grid spacing $)$ with 60 uniformly spaced vertical levels (in height coordinates) for 12 days. For models using height levels, a model top of $z_{\text {top }}=12000 \mathrm{~m}$ is suggested, which leads to a vertical grid spacing of $\Delta z=200 \mathrm{~m}$. This means that the model interfaces are positioned at $0,200,400 \mathrm{~m}$, etc., and that the full model levels are placed at 100, 300, $500 \mathrm{~m}$, etc. From (6), the height position of the model top corresponds to $p_{\text {top }} \approx 254.944 \mathrm{hPa}$. Information on the placement of vertical levels when hybrid coefficients are used is discussed in Appendix B.

Normalized $\ell_{1}, \ell_{2}$ and $\ell_{\infty}$ error norms $((14)-(16))$ should be computed for all tracers at $t=12$ days against the initial conditions. For test $1-1$, we have specified a tracer field $q_{1}(\lambda, \varphi, z)$ and a correlated field $q_{2}(\lambda, \varphi, z)$. We define the correlation plot of $q_{1}$ and $q_{2}$ as the scatter plot obtained from plotting the mixing ratios $\left(q_{1}\right)_{k}$ against $\left(q_{2}\right)_{k}$ for each cell $k$. For the given distribution, one will initially obtain the quadratic curve given by (32). As the simulation progresses, the nonlinear correlation between these tracers will be lost due to numerical errors and so the scatter plot will drift from its initial distribution. The correlation plot at $t=6$ days, the point of maximum deformation, reveals important information on how well the scheme preserves these correlations. Lauritzen and Thuburn (2012) define three categories of numerical mixing: real mixing, $\ell_{\mathrm{r}}$, where the numerical mixing resembles physical mixing; rangepreserving unmixing, $\ell_{\mathrm{u}}$, where the numerical unmixing is within the initial data range; and overshooting, $\ell_{0}$, numerical unmixing that falls outside the initial data range. These mixing diagnostics $\ell_{\mathrm{r}}, \ell_{\mathrm{u}}$ and $\ell_{\mathrm{o}}$, given in Appendix $\mathrm{C}$, should be computed for $q_{1}$ and $q_{2}$ at $t=6$ days. These mixing diagnostics should only be calculated for the 5 levels surrounding (and including) the 

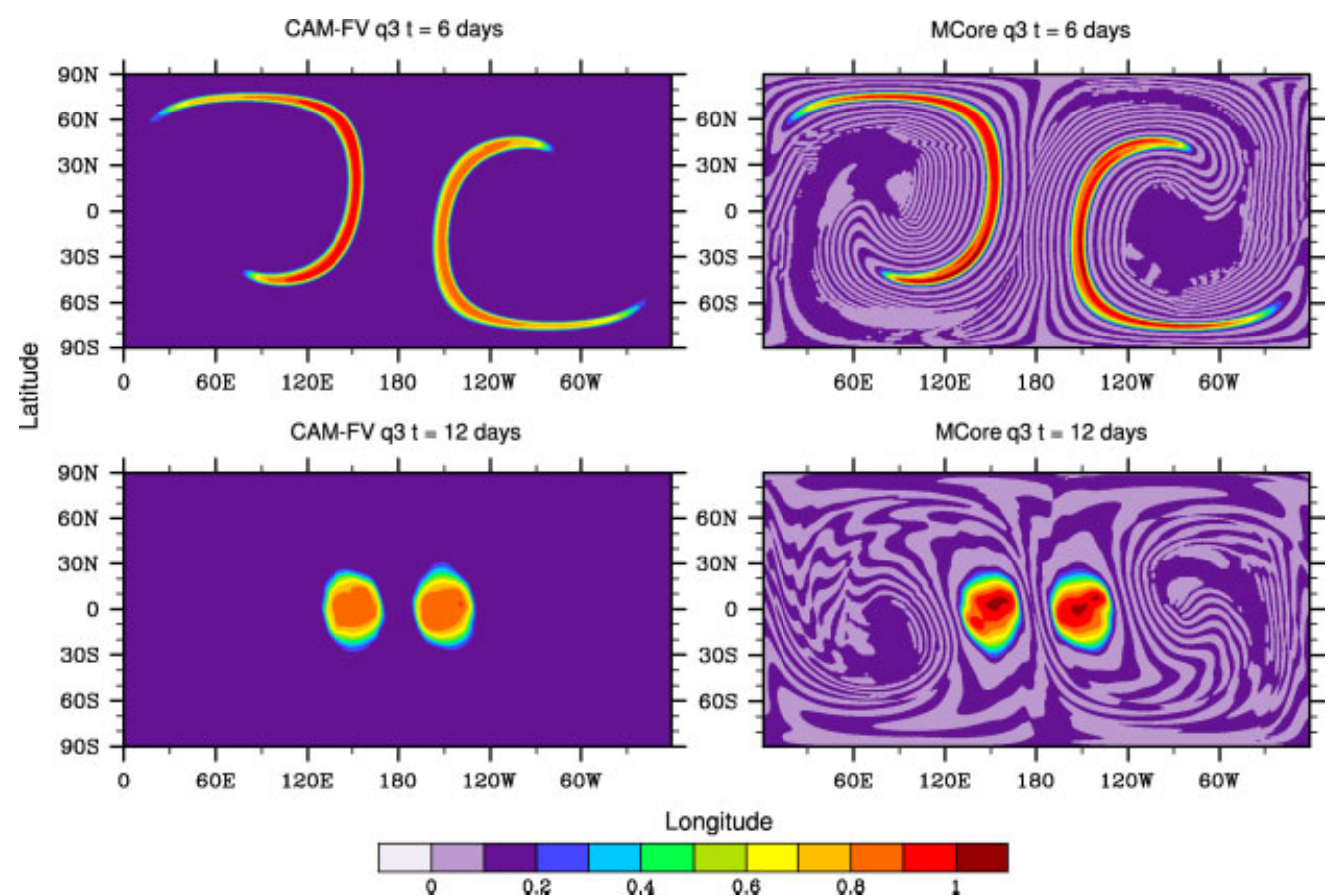

Figure 3. Test 1-1: latitude-longitude plots of tracer $q_{3}$ at height $4900 \mathrm{~m}$ and time $t=6$ days (top) and $t=12$ days (bottom) for CAM-FV (left) and MCore (right). The resolution is $1^{\circ} \times 1^{\circ}$ with 60 vertical levels. This figure is available in colour online at wileyonlinelibrary.com/journal/qj

Table 3. Test 1-1: normalized error norms for the tracers and for the sum $(3 / 10)\left(q_{1}+q_{2}+q_{3}\right)+q_{4}$ at $t=12$ days.

\begin{tabular}{lcccccc}
\hline & & $q_{1}$ & $q_{2}$ & $q_{3}$ & $\frac{3}{10}\left(q_{1}+q_{2}+q_{3}\right)+q_{4}$ \\
\hline CAM-FV & $\ell_{1}$ & 0.1210 & 0.0005 & 0.0236 & 0.0011 \\
& $\ell_{2}$ & 0.0998 & 0.0056 & 0.2519 & 0.0001 \\
MCore & $\ell_{\infty}$ & 0.1923 & 0.1967 & 0.8589 & 0.00130 \\
& $\ell_{1}$ & 0.1774 & 0.0009 & 0.0251 & 0.3990 & 0.0014 \\
& $\ell_{2}$ & 0.1552 & 0.0071 & 0.2354 & 0.0125 \\
& $\ell_{\infty}$ & 0.3384 & 0.2629 & 0.8444 & 0.0403 & 0.0003 \\
\end{tabular}

$4900 \mathrm{~m}$ vertical level; this is to improve computational efficiency. The mixing diagnostics are described in detail by Lauritzen and Thuburn (2012).

The final diagnostic concerns the ability of the transport scheme to maintain the sum of tracers. The tracer $q_{4}$ is designed such that the sum of $q_{4}$ and the other tracer fields with weight $(3 / 10)$ is equal to 1 . Normalized $\ell_{1}, \ell_{2}$ and $\ell_{\infty}$ error norms should be computed for this sum against the constant 1 . These error norms can be calculated at any time of the simulation, as the sum should equal 1 for all time.

\subsection{Example results}

We present example results for test 1-1 using two dynamical cores: CAM-FV and MCore (see Section 2.1). These results are used to illustrate the characteristics of the test case and not for the purpose of model intercomparison. Figure 3 shows the tracer $q_{3}$ at time $t=6$ and 12 days for both CAM-FV and MCore for test $1-1$. The plots are taken at the $4900 \mathrm{~m}$ height level and the resolution is $1^{\circ} \times 1^{\circ}$ with 60 vertical levels. The plot at $t=6$ days shows the extent of the flow deformation. Note that the results for MCore have been interpolated from its native cubed-sphere grid to the latitude-longitude grid. MCore makes use of a positivity filter but does not use a monotonic filter and so over- and undershoots are observed in the tracer field (which has a global background value of 0.1). The tracer-transport algorithm in CAM-FV is almost monotonic, as the dimensional splitting of the limiter allows the violation of monotonicity, and therefore any over- or undershoots are smaller in magnitude than in MCore. At the $4900 \mathrm{~m}$ height level there is no over- or undershooting for CAM-FV.

The tracers at the final time, $t=12$ days, can be compared with the initial conditions shown in Figure 2. The normalized error norms for test 1-1 are given in Table 3. These error norms allow us to assess how well a transport scheme can advect smooth data, tracer $q_{1}$, and how well the transport scheme can maintain the steep gradients of the discontinuous tracer, $q_{3}$. The final column of Table 3 provides the normalized error norms for the sum $(3 / 10)\left(q_{1}+q_{2}+q_{3}\right)+q_{4}$ against the constant 1 at time $t=12$ days. Although the values are orders of magnitude smaller than the error norms for the individual tracers, these error norms show that both models are unable to maintain the linear sum of four tracers properly for the duration of the simulation.

The left plot of Figure 4 shows a schematic taken from Lauritzen and Thuburn (2012) to demonstrate where real mixing, rangepreserving unmixing and overshooting occurs on the correlation plots. For CAM-FV and MCore, the correlation plots are shown in the centre and right plots of Figure 4 and the mixing diagnostics are shown in Table 4. The horizontal lines on the correlation plots show the initial maximum and minimum values of the tracer $q_{2}$, the quadratic curve shows the initial correlation between $q_{1}$ and $q_{2}$ and the diagonal line boxes in the real mixing convex hull. The mixing diagnostics and correlation plots show that there is overshooting with MCore, yet no overshooting with CAMFV (note that, although there is no overshooting for this test, overshooting does occur for CAM-FV with tracer $q_{3}$ ). The mixing diagnostics show that MCore produces more real mixing and more unmixing than CAM-FV.

\subsection{Comparison with two-dimensional tests}

To highlight the importance of three-dimensional testing, we compare the example results with those from the two-dimensional test, test case 4, of Nair and Lauritzen (2010). To ensure a fair 

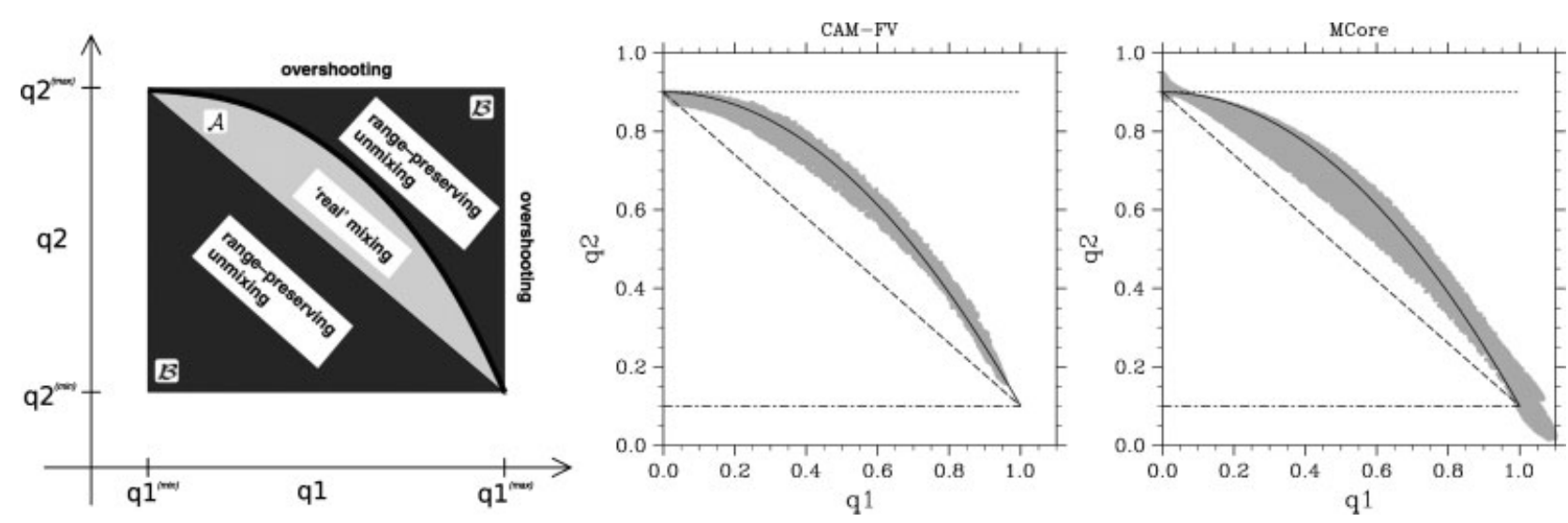

Figure 4. The left plot shows a schematic of the classification of numerical mixing (reproduced from Lauritzen and Thuburn (2012) with permission of the Royal Meteorological Society). The centre and right plots are the correlation plots of $q_{1}$ against $q_{2}$ for the five levels surrounding $4900 \mathrm{~m}$ at time $t=6$ days for CAM-FV (centre) and MCore (right) for test $1-1$.

Table 4. Test 1-1: mixing diagnostics. Real mixing, $\ell_{\mathrm{r}}$; range-preserving unmixing, $\ell_{\mathrm{u}} ;$ overshooting, $\ell_{\mathrm{o}}$.

\begin{tabular}{lccc}
\hline & $\ell_{\mathrm{r}}$ & $\ell_{\mathrm{u}}$ & $\ell_{\mathrm{o}}$ \\
\hline CAM-FV & $1.04 \times 10^{-3}$ & $2.86 \times 10^{-4}$ & 0.0 \\
MCore & $2.53 \times 10^{-3}$ & $5.60 \times 10^{-4}$ & $1.08 \times 10^{-3}$ \\
\hline
\end{tabular}

comparison, we use the three-dimensional tracers defined in our article for test $1-1$ and the velocities $u=u_{\mathrm{a}}, v=v_{\mathrm{a}}$ and $w=0$.

The three-dimensional test provides a challenging assessment of three-dimensional transport, in which it assesses both the horizontal and vertical components and also the coupling of the horizontal and vertical in the model framework. For example, for CAM-FV there are larger over- and undershoots for the twodimensional version of the test than for the full three-dimensional test. This shows the effects of diffusion from the vertical remapping in CAM-FV coupled with diffusion from the flux limiters in the horizontal discretization.

Error-norm analysis shows that the full three-dimensional test is more challenging than the two-dimensional version. The normalized error norms for each tracer after 12 days are larger with the three-dimensional version of the test. For example, for $q_{1}$ the normalized $\ell_{1}, \ell_{2}$ and $\ell_{\infty}$ error norms for CAM-FV are $0.0849,0.0728$ and 0.1379 respectively for the two-dimensional flow, compared with $0.1210,0.0998$ and 0.1923 for the full threedimensional flow. Similarly, for MCore the normalized $\ell_{1}, \ell_{2}$ and $\ell_{\infty}$ error norms are $0.0909,0.0798$ and 0.1517 respectively for the two-dimensional flow, compared with $0.1774,0.1552$ and 0.3384 for the full three-dimensional flow.

The three-dimensional test also has an effect on the mixing diagnostics. For CAM-FV the real mixing is comparable, yet there is more range-preserving unmixing for the three-dimensional test than the two-dimensional test. This implies that the impact of the vertical Lagrangian coordinate coupled with the horizontal discretization in CAM-FV is to produce unphysical mixing. For MCore, the mixing diagnostics for the two-dimensional flow are all less than the corresponding diagnostic for the threedimensional test, indicating that the addition of the vertical discretization introduces both real and unphysical mixing and is a cause of overshooting. For both dynamical cores, the twodimensional test preserves the sum of the tracers better than the three-dimensional test.

\section{Test 1-2: Hadley-like meridional circulation}

The emphasis of the second test is on horizontal-vertical coupling. Many transport algorithms in dynamical cores are horizontally-vertically split and it is important to understand how much effect this splitting has on the accuracy of the scheme. The prescribed flow is designed to contain a number
Table 5. List of constants used for the three-dimensional Hadley-like meridional circulation test case (test $1-2$ ).

\begin{tabular}{lll}
\hline Constant & Value & Description \\
\hline$\tau$ & $86400 \mathrm{~s}$ & Period of motion (here 1 day) \\
$K$ & 5 & Number of overturning cells \\
$u_{0}$ & $40 \mathrm{~m} \mathrm{~s}^{-1}$ & Reference zonal velocity \\
$w_{0}$ & $0.15 \mathrm{~m} \mathrm{~s}^{-1}$ & Reference vertical velocity \\
$z_{1}$ & $2000 \mathrm{~m}$ & Lower boundary of tracer layer \\
$z_{2}$ & $5000 \mathrm{~m}$ & Upper boundary of tracer layer \\
\hline
\end{tabular}

of circulations, similar to the test given in Zerroukat and Allen (2012); an important difference here is that we reverse the flow to give an analytical solution. The list of constants used in test 1-2 is given in Table 5 .

The zonal, meridional and vertical velocity field for this test is specified as

$$
\begin{aligned}
u(\lambda, \varphi, z, t)= & u_{0} \cos (\varphi) \\
v(\lambda, \varphi, z, t)= & -\frac{a w_{0} \pi \rho_{0}}{K z_{\mathrm{top}} \rho} \cos (\varphi) \sin (K \varphi) \\
& \times \cos \left(\frac{\pi z}{z_{\mathrm{top}}}\right) \cos \left(\frac{\pi t}{\tau}\right) \\
w(\lambda, \varphi, z, t)= & \frac{w_{0} \rho_{0}}{K \rho}[-2 \sin (K \varphi) \sin (\varphi) \\
& +K \cos (\varphi) \cos (K \varphi)] \sin \left(\frac{\pi z}{z_{\mathrm{top}}}\right) \cos \left(\frac{\pi t}{\tau}\right)
\end{aligned}
$$

where the density equation (10) is used in the formulation of the meridional velocity $v$ and the vertical velocity $w$ (for heightbased coordinates). The symbol $\rho_{0}$ denotes the density at the surface, with $\rho_{0}=p_{0} /\left(R_{\mathrm{d}} T_{0}\right)$. The surface pressure is constant, with $p_{\mathrm{s}}(\lambda, \varphi)=p_{0}$. Since the pressure field $p$ varies neither in time nor in the horizontal direction, the vertical pressure velocity $\omega$ for pressure-based coordinates is easily obtained from (6), (28) and (38):

$$
\omega(\lambda, \varphi, p, t)=-g \rho w[\lambda, \varphi, z(p), t] .
$$

The density $\rho$ vanishes in this equation when plugging in (38). The density $\rho$ is time-independent and needs to be kept constant for advection schemes in conservation form. This design guarantees that this test is equivalent for tracer advection schemes written in both advective or conservation forms.

The vertical velocities for models with vertical $\sigma$ or hybrid $\sigma$-pressure $(\eta)$ coordinates are given by

$$
\dot{\eta}(\lambda, \varphi, \eta, t)=\dot{\sigma}(\lambda, \varphi, \sigma, t)=-\frac{g \rho}{p_{0}} w[\lambda, \varphi, z(p), t],
$$



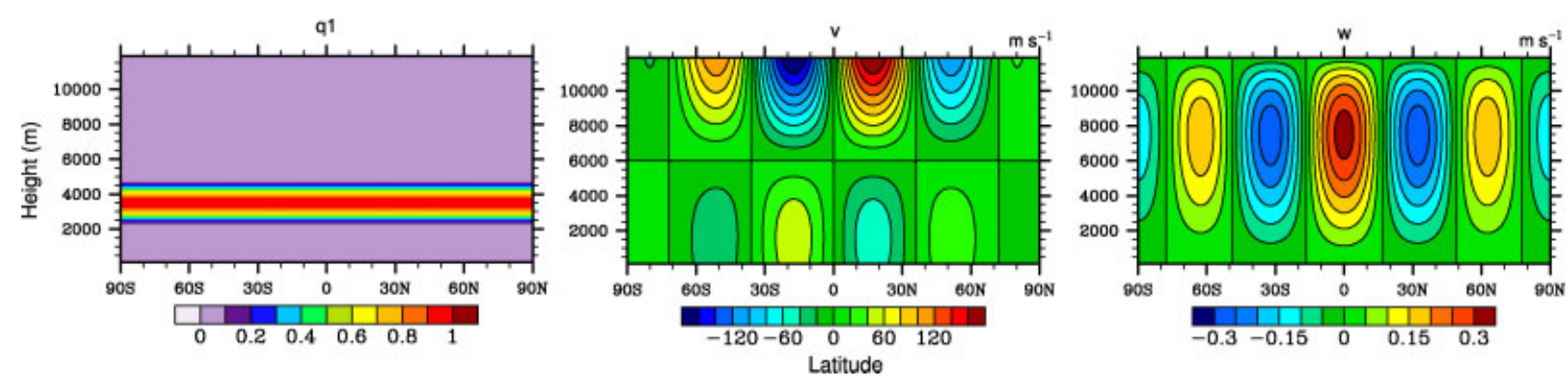

Figure 5. Test 1-2 initial conditions: latitude-height cross-section at $\lambda=180^{\circ}$ of tracer $q_{1}$ and velocities $v$ and $w$. This figure is available in colour online at wileyonlinelibrary.com/journal/qj

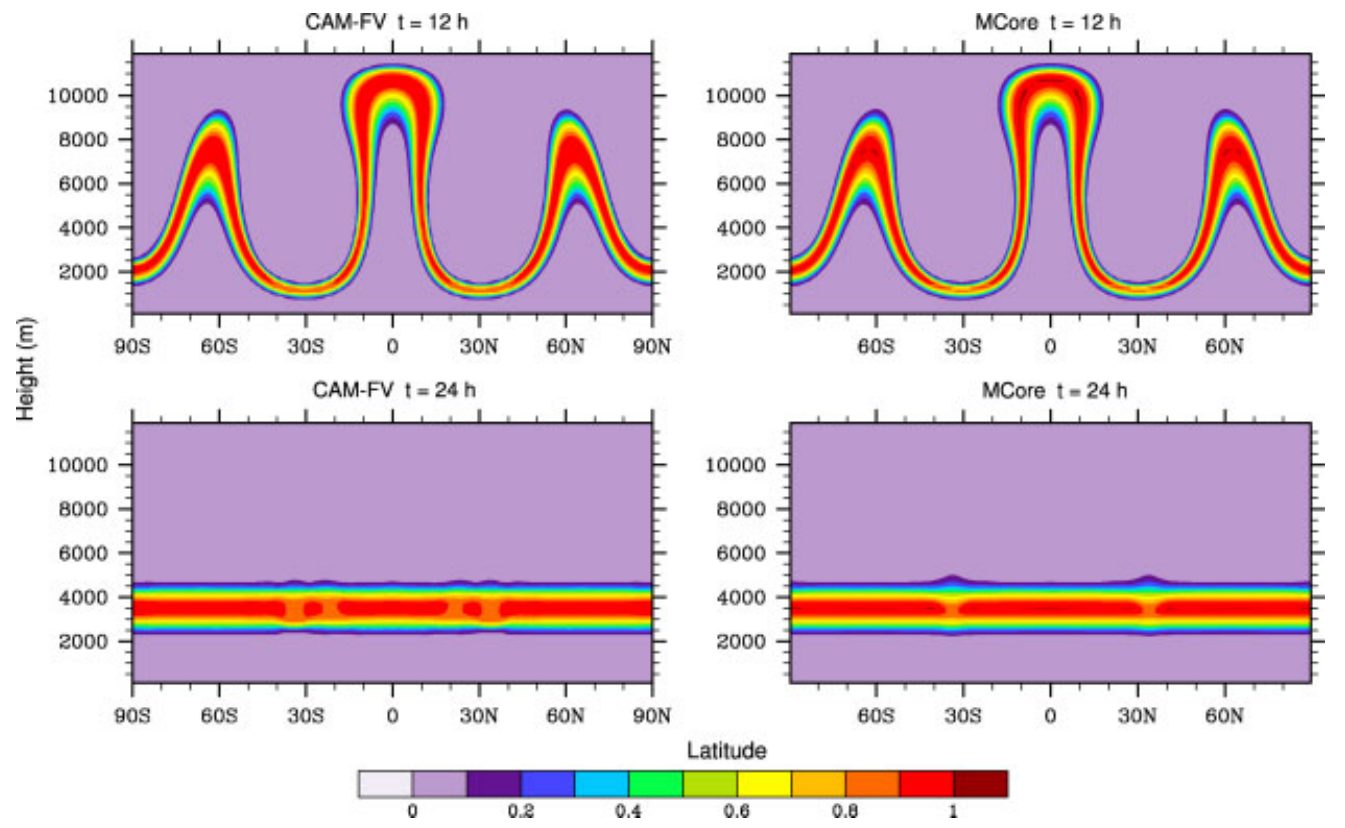

Figure 6. Test 1-2: latitude-height plots at $\lambda=180^{\circ}$ of tracer $q_{1}$ at time $t=12 \mathrm{~h}$ (top) and $t=24 \mathrm{~h}$ (bottom) for CAM-FV (left) and MCore (right). The resolution is $1^{\circ} \times 1^{\circ}$ with 60 vertical levels. This figure is available in colour online at wileyonlinelibrary.com/journal/qj

where $\eta$ and $\sigma$ are given by $\eta=\sigma=p / p_{0}$ due to the choice of constant surface pressure, $p_{\mathrm{s}}=p_{0}$. As in test $1-1$, note that this formulation assumes that the reference pressure for the hybrid $\eta$ coordinate is set to $p_{0}=1000 \mathrm{hPa}$. If a floating Lagrangian coordinate is used on the basis of varying pressure thicknesses, we recommend a mechanism that utilizes (39) in combination with the discrete approach described in Appendix A.

The surface is flat with $z_{\mathrm{s}}=0 \mathrm{~m}$, or equivalently surface geopotential $\Phi_{s}=0 \mathrm{~m}^{2} \mathrm{~s}^{-2}$. The tracer field consists of a vertical layer that is deformed over the duration of the simulation. It is given by

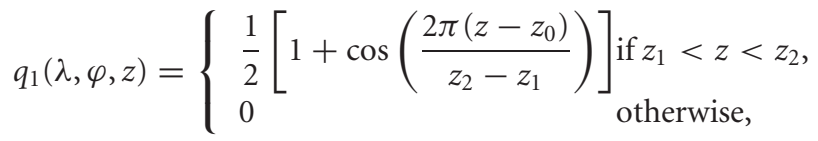

where $z_{0}=\frac{1}{2}\left(z_{1}+z_{2}\right)$. For models with pressure-based coordinates, (6) and (7) need to be used to convert between height and pressure positions. Figure 5 shows latitude-height cross-sections of the initial velocities $v$ and $w$ and the initial tracer $q_{1}$.

\subsection{Grid spacings and diagnostics}

This test should be run at $2^{\circ} \times 2^{\circ}$ resolution with 30 uniformly spaced vertical levels, $1^{\circ} \times 1^{\circ}$ resolution with 60 uniformly spaced vertical levels and $0.5^{\circ} \times 0.5^{\circ}$ resolution with 120 uniformly spaced vertical levels. For models using height levels, a maximum altitude of $z_{\text {top }}=12000 \mathrm{~m}$ is suggested. These resolutions correspond to an approximate horizontal grid spacing of about
220,110 and $55 \mathrm{~km}$ with a vertical grid spacing of $\Delta z=400,200$ and $100 \mathrm{~m}$, respectively. From (6), the position of the model top yields the pressure $p_{\text {top }} \approx 254.944 \mathrm{hPa}$. For 60 vertical levels, the model interfaces are positioned at $0,200,400 \mathrm{~m}$, etc., and the full model levels are placed at 100, 300, $500 \mathrm{~m}$, etc., as with test $1-1$.

The simulation is run for $t=1$ day, until the tracer field returns to its original configuration. For each resolution, normalized error norms $\ell_{1}, \ell_{2}$ and $\ell_{\infty}$ should be computed by comparing the results at $t=1$ day against the initial configuration. This will allow convergence rates to be calculated to assess the numerical order of accuracy of the tracer-transport algorithm.

\subsection{Example results}

Figure 6 shows the tracer $q_{1}$ at time $t=12 \mathrm{~h}$ and $t=24 \mathrm{~h}$ for test $1-2$ when using $1^{\circ} \times 1^{\circ}$ resolution with 60 vertical levels. Again, the results from MCore are interpolated from its native cubed-sphere grid to the latitude-longitude grid. The results for both CAM-FV and MCore are shown as latitude-height crosssections at the longitude $\lambda=180^{\circ}$. The plot demonstrates how the tracer is deformed by the flow field and how it returns to its initial state after $24 \mathrm{~h}$. Both models have produced 'gaps' in the final tracer at approximately $30^{\circ} \mathrm{N}$ and $30^{\circ} \mathrm{S}$. This is due to the extreme stretching that takes place in this area of the tracer and it can be seen in the tracer plots at $12 \mathrm{~h}$. Overshoots are evident for MCore at both 12 and $24 \mathrm{~h}$.

Example normalized error norms for CAM-FV and MCore are given in Table 6 for test $1-2$. Also shown are the average convergence rates for each error norm. 
Table 6. Test 1-2: normalized error norms at different resolutions and the average convergence rate for each error norm.

\begin{tabular}{|c|c|c|c|c|c|}
\hline & & $2^{\circ} L 30$ & $1^{\circ} L 60$ & $1 / 2^{\circ} L 120$ & Convergence \\
\hline \multirow[t]{3}{*}{ CAM-FV } & $\ell_{1}$ & 0.1810 & 0.0411 & 0.0124 & 1.93 \\
\hline & $\ell_{2}$ & 0.2047 & 0.0536 & 0.0159 & 1.84 \\
\hline & $\ell_{\infty}$ & 0.4705 & 0.1575 & 0.0473 & 1.66 \\
\hline \multirow[t]{3}{*}{ MCore } & $\ell_{1}$ & 0.1368 & 0.0286 & 0.0063 & 2.22 \\
\hline & $\ell_{2}$ & 0.1659 & 0.0462 & 0.0113 & 1.94 \\
\hline & $\ell_{\infty}$ & 0.4214 & 0.1586 & 0.0435 & 1.64 \\
\hline
\end{tabular}

Table 7. List of constants used for the horizontal advection of thin cloud-like tracers in the presence of the orography test case (test 1-3).

\begin{tabular}{lll}
\hline Constant & Value & Description \\
\hline$u_{0}$ & $2 \pi a / \tau$ & Maximum wind speed \\
$\tau$ & $1036800 \mathrm{~s}$ & Period of motion (here 12 days) \\
$\alpha$ & $\pi / 6$ & Rotation angle (radians, $30^{\circ}$ ) \\
$\lambda_{\mathrm{m}}$ & $3 \pi / 2$ & Mountain longitude centre point \\
$\varphi_{\mathrm{m}}$ & 0 & Mountain latitude centre point \\
$h_{0}$ & $2000 \mathrm{~m}$ & Maximum mountain height \\
$R_{\mathrm{m}}$ & $3 \pi / 4$ & Mountain radius (radians) \\
$\zeta_{\mathrm{m}}$ & $\pi / 16$ & Mountain oscillation half-width (radians) \\
$\lambda_{\mathrm{p}}$ & $\pi / 2$ & Cloud-like tracer longitude centre point \\
$\varphi_{\mathrm{p}}$ & 0 & Cloud-like tracer latitude centre point \\
$z_{p, 1}$ & $3050 \mathrm{~m}$ & First cloud-like tracer altitude \\
$z_{p, 2}$ & $5050 \mathrm{~m}$ & Second cloud-like tracer altitude \\
$z_{p, 3}$ & $8200 \mathrm{~m}$ & Third cloud-like tracer altitude \\
$\Delta z_{p, 1}$ & $1000 \mathrm{~m}$ & First cloud-like tracer thickness \\
$\Delta z_{p, 2}$ & $1000 \mathrm{~m}$ & Second cloud-like tracer thickness \\
$\Delta z_{p, 3}$ & $400 \mathrm{~m}$ & Third cloud-like tracer thickness \\
$R_{\mathrm{p}}$ & $\pi / 4$ & Cloud-like deck radius (radians) \\
\hline
\end{tabular}

\section{Test 1-3: horizontal advection of thin cloud-like tracers in the presence of orography}

The third test case investigates the ability of the tracer-transport algorithm to advect tracers accurately over orography. For models that utilize terrain-following coordinates, the orography ensures that the tracer is transported between model levels. The list of constants used in test $1-3$ is given in Table 7 .

For this test the zonal, meridional and vertical velocity fields along surfaces of constant height (above the mean sea level) are specified as

$$
\begin{aligned}
u(\lambda, \varphi, z, t) & =u_{0}(\cos \varphi \cos \alpha+\sin \varphi \cos \lambda \sin \alpha), \\
v(\lambda, \varphi, z, t) & =-u_{0} \sin \lambda \sin \alpha, \\
w(\lambda, \varphi, z, t) & =0
\end{aligned}
$$

where $\alpha$ is a rotation angle. The velocity field transports the tracers horizontally (at a constant height) once around the sphere over a duration of 12 days. Note that some models will require $u=v=0$ for $z<h_{0}$ to prevent problems occurring due to flow below the maximum height of the orography. This is a valid modification of the initial condition that will not impact the characteristics of the tracer-transport test. The surface elevation is a three-dimensional variant of a Schär-like (Schär et al., 2002) mountain with compact support, centred around the centre point $\left(\lambda_{\mathrm{m}}, \varphi_{\mathrm{m}}\right)$. The great circle distance from the mountain centre point (in radians) is defined as

$$
r_{\mathrm{m}}(\lambda, \varphi)=\arccos \left[\sin \varphi_{\mathrm{m}} \sin \varphi+\cos \varphi_{\mathrm{m}} \cos \varphi \cos \left(\lambda-\lambda_{\mathrm{m}}\right)\right]
$$

The surface elevation is then given by

$$
z_{\mathrm{s}}(\lambda, \varphi)=\left\{\begin{aligned}
\frac{h_{0}}{2}\left[1+\cos \left(\frac{\pi r_{\mathrm{m}}}{R_{\mathrm{m}}}\right)\right] \cos ^{2}\left(\frac{\pi r_{\mathrm{m}}}{\zeta_{\mathrm{m}}}\right), & \text { if } r_{\mathrm{m}}<R_{\mathrm{m}}, \\
0, & \text { otherwise. }
\end{aligned}\right.
$$

This choice ensures that the topography is flat away from the mountain but oscillates strongly over the mountain range itself. The surface geopotential is then given by $\Phi_{s}(\lambda, \varphi)=g z_{s}(\lambda, \varphi)$. The surface pressure is obtained by substituting $z=z_{\mathrm{s}}(\lambda, \varphi)$ in (6). The surface height, surface geopotential and horizontal velocities are shown in Figure 7.

Three thin cloud-like passive tracers are defined to represent lower-level, medium-level and upper-level cloud decks. These three cloud-like layers are initially placed away from the mountain in a region of flat topography so as to evaluate error norms more easily after one revolution around the sphere. The lateral great circle distance from the cloud centre point (in radians) is defined as

$$
r_{\mathrm{p}}(\lambda, \varphi)=\arccos \left[\sin \varphi_{\mathrm{p}} \sin \varphi+\cos \varphi_{\mathrm{p}} \cos \varphi \cos \left(\lambda-\lambda_{\mathrm{p}}\right)\right]
$$

Similarly we define a vertical distance from the centre of each cloud level,

$$
r_{z, i}(z)=\left|z-z_{\mathrm{p}, i}\right|
$$

where $i \in\{1,2,3\}$. If pressure-based vertical coordinates are used, the height $z(p)$ needs to be computed according to (7) first before applying (48). The lower-level and medium-level cloud-like tracers are disc-shaped, with the three-dimensional mixing ratio

$$
\begin{aligned}
& q_{i}(\lambda, \varphi, z) \\
& =\left\{\begin{array}{c}
\frac{1}{4}\left[1+\cos \left(\frac{2 \pi r_{z, i}(z)}{\Delta z_{\mathrm{p}, i}}\right)\right]\left[1+\cos \left(\frac{\pi r_{\mathrm{p}}(\lambda, \varphi)}{R_{\mathrm{p}}}\right)\right], \\
\text { if } r_{z, i}(z)<\frac{1}{2} \Delta z_{\mathrm{p}, i} \text { and } r_{\mathrm{p}}(\lambda, \varphi)<R_{\mathrm{p}}, \\
0, \quad \text { otherwise, }
\end{array}\right.
\end{aligned}
$$

for $i \in\{1,2\}$. The upper-level cloud-like tracer is box-shaped, with mixing ratio

$$
q_{3}(\lambda, \varphi, z)= \begin{cases}1, & \text { if } r_{z, 3}(z)<\frac{1}{2} \Delta z_{\mathrm{p}, 3} \text { and } r_{\mathrm{p}}(\lambda, \varphi)<R_{\mathrm{p}} \\ 0, & \text { otherwise. }\end{cases}
$$

The total tracer field, $q_{4}$, is the sum of these three cloud-like tracers:

$$
q_{4}(\lambda, \varphi, z)=q_{1}(\lambda, \varphi, z)+q_{2}(\lambda, \varphi, z)+q_{3}(\lambda, \varphi, z) .
$$

Selected cross-sections of the four tracers are shown in Figure 8. 

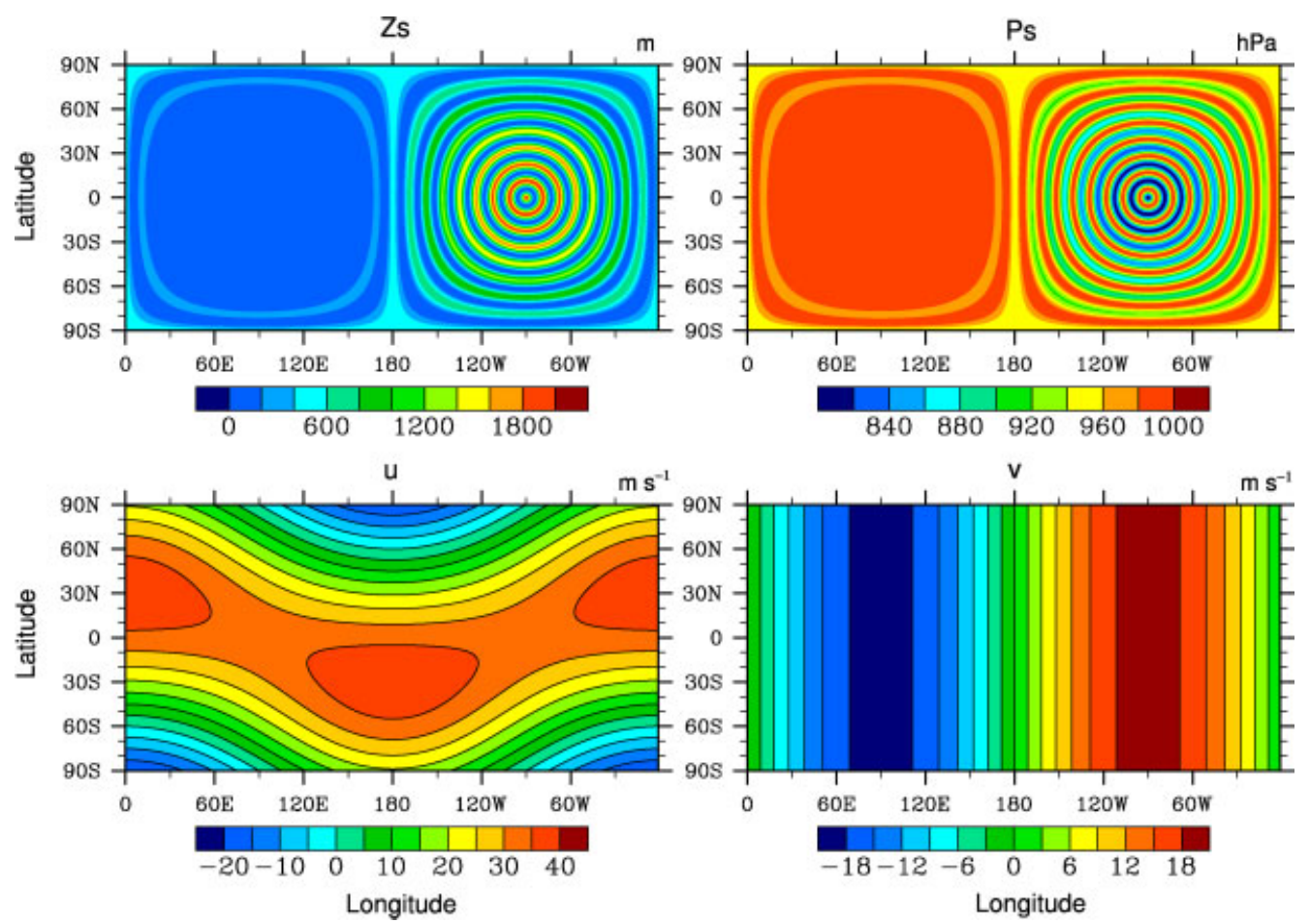

Figure 7. Test 1-3 initial conditions: latitude-longitude plots of surface height $z_{\mathrm{s}}$ (top left), surface pressure $p_{\mathrm{s}}$ (top right), zonal velocity $u$ (bottom left) and meridional velocity $v$ (bottom right). This figure is available in colour online at wileyonlinelibrary.com/journal/qj
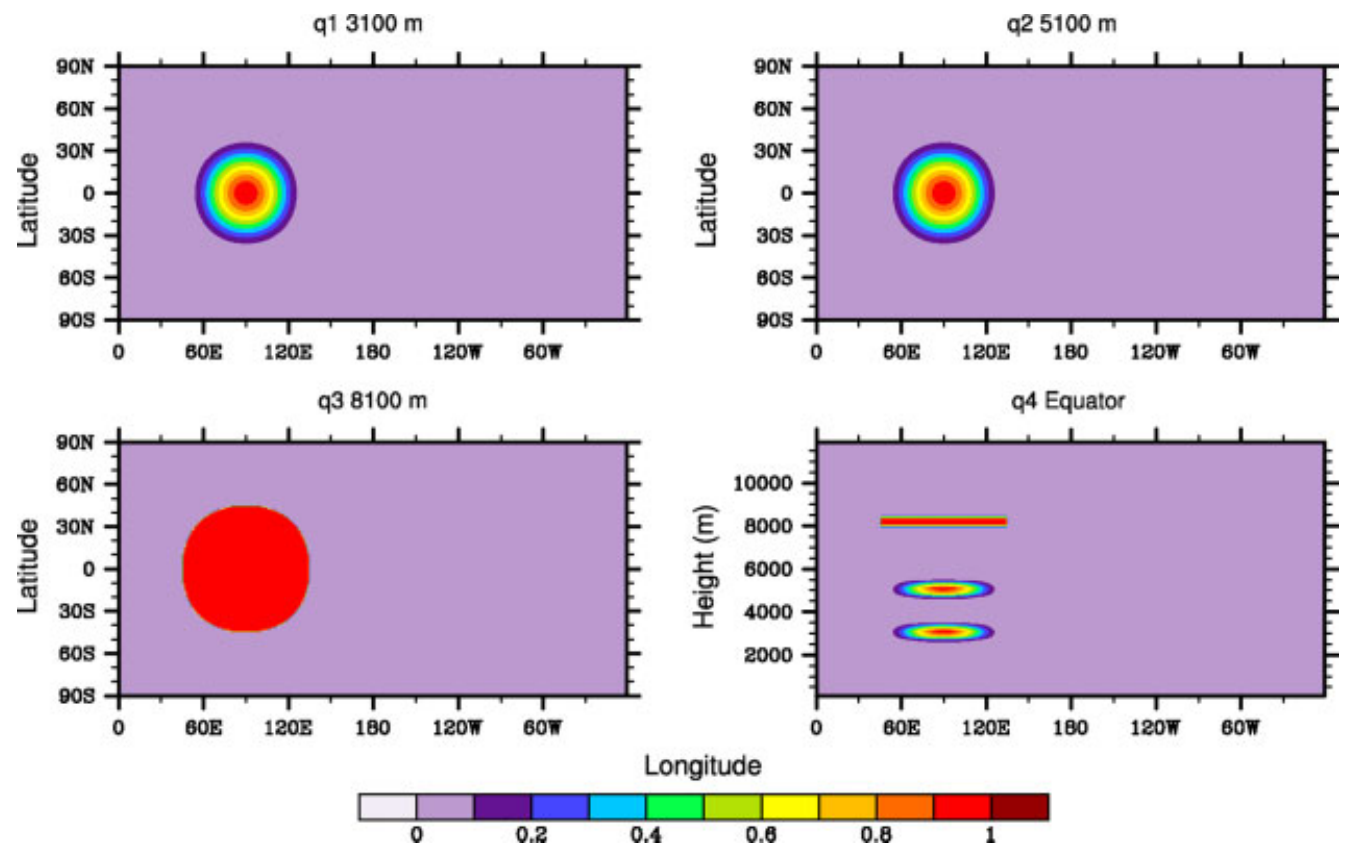

Figure 8. Test 1-3 initial conditions: latitude-longitude plots of tracer $q_{1}$ at $3100 \mathrm{~m}$ (top left), $q_{2}$ at $5100 \mathrm{~m}$ (top right) and $q_{3}$ at $8100 \mathrm{~m}$ (bottom left) and a longitude-height cross-section along the Equator of tracer $q_{4}$. This figure is available in colour online at wileyonlinelibrary.com/journal/qj

\section{1. 'Perceived vertical velocity'}

If terrain-following coordinates are utilized, then the tracers should pass between vertical model levels to ensure that the physical vertical velocity $w$ is zero. However, due to the design of some models, it may not be possible to enforce no vertical velocity $(w=0)$ unless there is an explicitly prescribed 'perceived vertical velocity', i.e. a mechanism for enforcing exchange between vertically stacked model levels. Without this, the tracer would be advected along a sloping model level, which is different from purely horizontal transport at constant height. To run test 1-3 correctly, this means that a non-zero imposed 'vertical velocity' must be applied in the presence of topography for these models. This perceived vertical velocity reflects that the terrain-following coordinate surfaces slope up- and downwards.
The vertical motion thereby ensures that there is an exchange of tracers between the sloping model levels in the case of purely horizontal advection. The perceived vertical velocity is derived in Appendix D.

\subsection{Grid spacings and diagnostics}

This test should be run at $1^{\circ} \times 1^{\circ}$ resolution $(\sim 110 \mathrm{~km}$ equatorial grid spacing) with 30,60 and 120 vertical levels for 12 days. For models using height levels, a model top of $z_{\text {top }}=12000 \mathrm{~m}$ is suggested, with a uniformly spaced vertical grid spacing of $\Delta z$ in the flat regions away from the mountain range. For the 60 vertical level set-up, this means that the model interfaces are positioned at $0,200,400 \mathrm{~m}$, etc., and that the full model levels are placed at $100,300,500 \mathrm{~m}$, etc., in the flat regions. If the model utilizes 

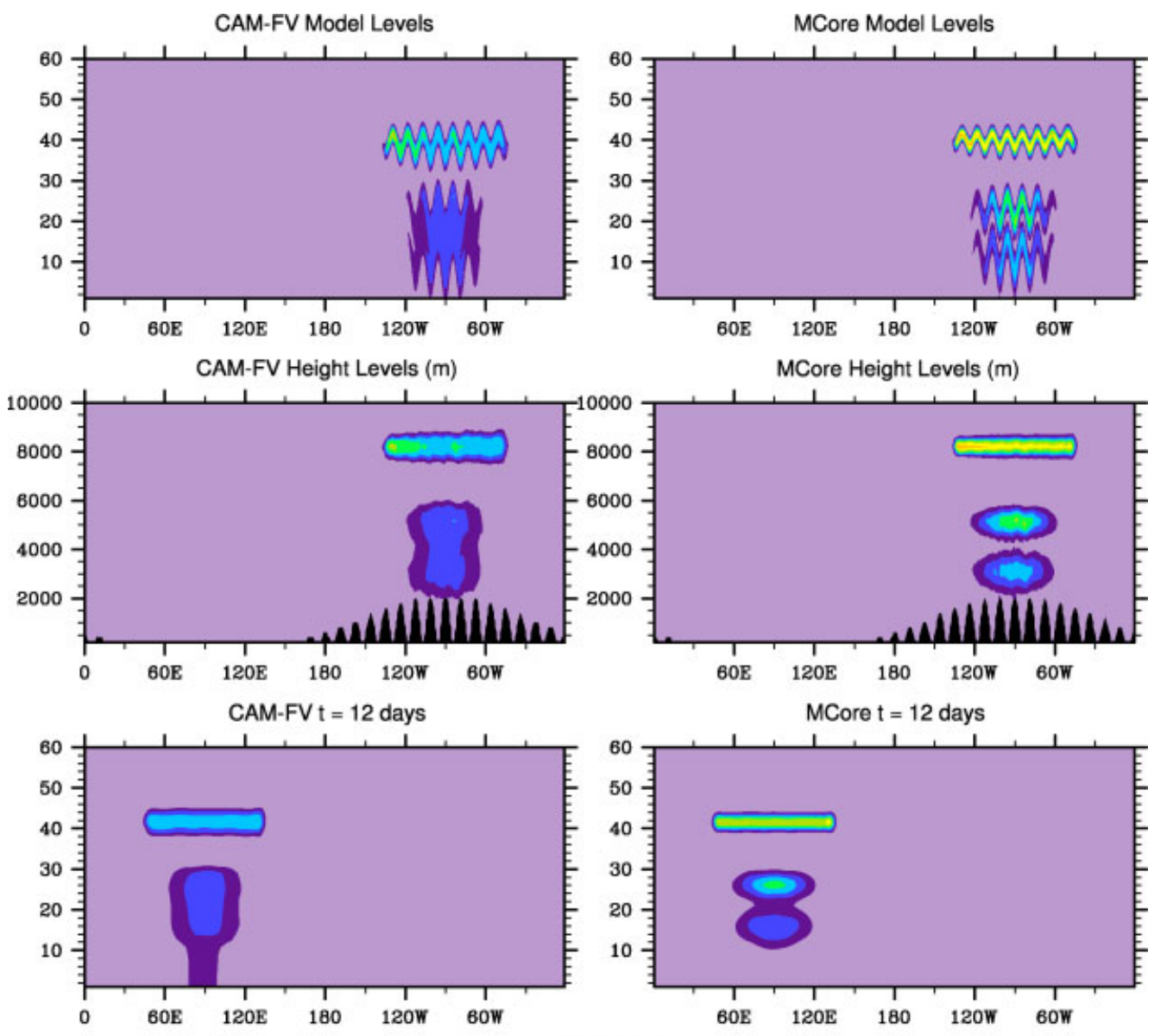

Longitude

Figure 9. Test 1-3: longitude-height cross-section of tracer $q_{4}$ at the Equator, on model levels (top) and interpolated to constant height levels (middle) at time $t=6$ days for CAM-FV (left) and MCore (right). The black shading indicates the mountain. The results at time $t=12$ days on model levels (bottom) are also shown. The resolution is $1^{\circ} \times 1^{\circ}$ with 60 vertical levels. This figure is available in colour online at wileyonlinelibrary.com/journal/qj

orography-following vertical coordinates then the grid spacing will be non-uniform over the mountain range. From (6) the height position of the model top corresponds to $p_{\text {top }} \approx 254.944 \mathrm{hPa}$.

Normalized $\ell_{1}, \ell_{2}$ and $\ell_{\infty}$ error norms should be computed for $q_{1}, q_{2}, q_{3}$ and $q_{4}$ at $t=12$ days against the initial conditions, for each of the vertical resolutions.

\subsection{Example results}

Cross-sections showing both longitude-model level and longitude-height levels are taken at the Equator for tracer $q_{4}$ on day 6 and are shown in the top and centre plots of Figure 9 for test $1-3$. The top plots show the tracer on model levels, while the middle plots show the tracer interpolated to constant height levels. Both CAM-FV and MCore use terrain-following vertical coordinates and therefore the tracer passes between model levels. Both models smooth out the tracer as it is passed between the model levels and this reduces the tracer maximum.

The bottom plot of Figure 9 shows the longitude-model level cross-section of tracer $q_{4}$ at time $t=12$ days. In the presence of strong diffusion, the two lower tracers can be merged into one (for example, with CAM-FV). The normalized error norms for test $1-3$ are given in Tables 8 and 9. Note that the error norms are calculated at time $t=12$ days and are only calculated in the western hemisphere; this is away from the mountain, where the vertical levels are equidistant in height. Both CAM-FV and MCore produce the smallest error norms for tracer $q_{2}$. This is because the vertical levels of the hybrid terrain-following coordinates become smoother and flatter with height and therefore there is less transfer between model levels for $q_{2}$ than for $q_{1}$. There is even less transfer between model levels for $q_{3}$ than $q_{2}$; however,
Table 8. Test 1-3: normalized error norms for tracers $q_{1}, q_{2}$ and $q_{3}$. The resolution is $1^{\circ} \times 1^{\circ} \mathrm{L} 60$.

\begin{tabular}{lcccc}
\hline & & $q_{1}$ & $q_{2}$ & $q_{3}$ \\
\hline CAM-FV & $\ell_{1}$ & 1.56 & 1.14 & 1.31 \\
& $\ell_{2}$ & 0.84 & 0.75 & 1.86 \\
\multirow{3}{*}{ MCore } & $\ell_{\infty}$ & 0.78 & 0.74 & 0.88 \\
& $\ell_{1}$ & 1.07 & 0.82 & 0.85 \\
& $\ell_{2}$ & 0.69 & 0.57 & 1.32 \\
& $\ell_{\infty}$ & 0.70 & 0.57 & 0.71 \\
\hline
\end{tabular}

Table 9. Test 1-3: tracer $q_{4}$ normalized error norms at different vertical resolutions (the horizontal resolution is $1^{\circ} \times 1^{\circ}$ ).

\begin{tabular}{lcccc}
\hline & & $L 30$ & $L 60$ & $L 120$ \\
\hline CAM-FV & $\ell_{1}$ & 1.35 & 1.33 & 1.31 \\
& $\ell_{2}$ & 0.81 & 0.77 & 0.78 \\
\multirow{3}{*}{ MCore } & $\ell_{\infty}$ & 0.88 & 0.85 & 0.91 \\
& $\ell_{1}$ & 1.08 & 0.89 & 0.83 \\
& $\ell_{2}$ & 0.70 & 0.57 & 0.55 \\
& $\ell_{\infty}$ & 0.81 & 0.71 & 0.73 \\
\hline
\end{tabular}

$q_{3}$ is discontinuous whereas $q_{2}$ is smooth. Table 9 shows that an increase in vertical resolution, while keeping the horizontal resolution constant, provides only a small improvement in the error norms. This result is due to the design of the test, as increasing the number of vertical levels leads to more interfaces that the tracer must pass through. 


\section{Conclusions}

This article has presented three tracer-transport test cases that can be easily incorporated into dynamical cores. The tests use prescribed non-divergent velocities and are designed so that the tracer returns to its initial position for straightforward comparison with an analytical solution. These tests help assess the ability of transport schemes to model three-dimensional tracer transport. The focus of the tests is on the properties that are relevant to tracer transport. These include physical properties, such as positivity and preservation of nonlinear tracer correlations, and numerical issues, such as horizontal-vertical coupling and the use of terrain-following vertical coordinates. We have provided recommended set-ups and diagnostics that aim to establish a standard for three-dimensional tracer-transport test cases on the sphere. FORTRAN initialization routines are provided in the Supporting Information.

We have demonstrated the test cases and produced example results using two dynamical cores: CAM-FV and MCore. Error norms and mixing diagnostics have been provided to allow easy comparison with future dynamical cores. The results highlight the extent of the deformation in test $1-1$ and test $1-2$ and how the simple horizontal advection of tracers over orography becomes a challenging test when hybrid terrain-following coordinates are used.

\section{A. Appendix: Vertical Lagrangian pressure-based coordinates}

If an advection scheme utilizes a floating Lagrangian coordinate without explicit vertical transport, as in Lin (2004), the conservation law for the advection takes the form

$$
\frac{\partial}{\partial t}(\Delta p q)+\nabla \cdot(\mathbf{u} \Delta p q)=0
$$

where $\mathbf{u}$ denotes the horizontal wind vector and $\Delta p$ the pressure thickness of the layers. The vertical transport then needs to be mimicked by a vertical remapping algorithm after the horizontal advection step. The following discrete algorithm is suggested to prescribe the time-dependent deforming pressure surfaces. First, we recommend calculating the pressure values $p\left(t_{2}\right)$ at the future time $t_{2}=t_{1}+\Delta t$, where $\Delta t$ symbolizes the time-step length and $t_{1}$ is the current time, counted in seconds since the start of the advection test. The new pressure values are then discretely given by

$$
p\left(t_{2}\right)=p\left(t_{1}\right)+\Delta t \omega\left(\lambda, \varphi, p, t_{1}+\frac{\Delta t}{2}\right)
$$

where a time-centred evaluation of the time-dependent expressions is selected. The time dependent $\Delta p$ variation can then be computed as the difference of the pressures at model interfaces at time $t_{2}$ and set back to its initial value as part of a remapping algorithm.

For example, using (19) the pressure for test $1-1$ is updated as

$$
\begin{aligned}
p\left(t_{2}\right)= & p\left(t_{1}\right)+\Delta t \omega_{0} \sin \left[\lambda-\frac{2 \pi}{\tau}\left(t_{1}+\frac{\Delta t}{2}\right)\right] \\
& \times \cos (\varphi) \cos \left[\frac{2 \pi}{\tau}\left(t_{1}+\frac{\Delta t}{2}\right)\right] s\left(p\left(t_{1}\right)\right) .
\end{aligned}
$$

\section{B. Appendix: Placement of vertical levels using hybrid coefficients}

The hybrid orography-following $\eta$ coordinate (Simmons and Burridge, 1981) comprises a pressure coordinate with a $\sigma=p / p_{\mathrm{s}}$ component. The pressure at vertical level $\eta$ is given by

$$
p(\lambda, \varphi, \eta, t)=a(\eta) p_{0}+b(\eta) p_{s}(\lambda, \varphi, t)
$$

where $a(\eta)$ and $b(\eta)$ are the hybrid coefficients and $\eta=p(z) / p_{\mathrm{s}}$ under the special condition that $p_{s}=p_{0}$. Note that we also use this set-up for test $1-3$, as away from the mountain the reference surface pressure is equal to $p_{0}$. For isothermal conditions, the vertical pressure profiles are given by (6) and this can be used to relate $\eta$ to $z$. The hybrid coefficients at interface levels are then calculated as in Laprise and Girard (1990):

$$
\begin{aligned}
& a(\eta)=\eta-b(\eta), \\
& b(\eta)=\frac{\eta-\eta_{\mathrm{top}}}{1-\eta_{\mathrm{top}}}
\end{aligned}
$$

where $\eta_{\text {top }}=p\left(z_{\text {top }}\right) / p_{\mathrm{s}}$. The hybrid coefficients at full model levels, with index $k$, are computed by the linear average of the interface levels:

$$
\begin{aligned}
& a_{k}=\frac{1}{2}\left(a_{k+1 / 2}+a_{k-1 / 2}\right), \\
& b_{k}=\frac{1}{2}\left(b_{k+1 / 2}+b_{k-1 / 2}\right) .
\end{aligned}
$$

Note that, in the discrete system, equations (B1) and (6) are only equal for the interface levels and not for the full model levels. This is due to the linear average used to calculate (B4) and (B5). However, this discrepancy is small and does not affect the set-up of the tracer tests in this article. Therefore, for the purpose of the tracer tests in this article, it can be assumed that (B1) and (6) are equal on both interface and model levels.

\section{Appendix: Mixing diagnostics}

A measure of the types of numerical mixing that occur during the simulation can be quantitatively obtained using mixing diagnostics. Following Lauritzen and Thuburn (2012) and Lauritzen et al. (2012), there are three categories of numerical mixing: real mixing, where scatter points move to the concave side of $\psi$; range-preserving unmixing, where scatter points move to the convex side of $\psi$ or below the convex hull but not outside the initial data range; and overshooting, where scatter points fall outside the initial data range.

We first define $\Delta A_{k}$ as the area of grid cell $k$ and $A$ as the total area of the domain. Further, we define $d_{k}$ as the normalized shortest distance between the point $\left(\chi_{k}, \xi_{k}\right)$ and the initial $[\chi, \psi(\chi)]$ correlation curve. For the initial distribution given in $(32), d_{k}$ is defined as

$$
d_{k}=L\left(\chi_{k}^{(\psi)}, \chi_{k}, \xi_{k}\right)
$$

where

$$
C\left(\chi_{k}, \xi_{k}\right)=\frac{1}{12}\left[432 \chi_{k}+6 \sqrt{750\left(2 \xi_{k}-1\right)^{3}+5184 \chi_{k}^{2}}\right]^{1 / 3}
$$

$$
\chi_{k}^{(\text {root })}\left(\chi_{k}, \xi_{k}\right)=C\left(\chi_{k}, \xi_{k}\right)+\frac{1}{C\left(\chi_{k}, \xi_{k}\right)}\left(\frac{5}{24}-\frac{5}{12} \xi_{k}\right)
$$

$$
\chi_{k}^{(\psi)}\left(\chi_{k}, \xi_{k}\right)=\min \left[\max \left(\chi^{(\min )}, \chi_{k}^{(\operatorname{root})}\left(\chi_{k}, \xi_{k}\right)\right), \chi^{(\max )}\right]
$$

and

$$
L\left(\chi, \chi_{k}, \xi_{k}\right)=\sqrt{\left(\frac{\chi_{k}-\chi}{\chi^{(\max )}-\chi^{(\min )}}\right)^{2}+\left(\frac{\xi_{k}-\psi(\chi)}{\xi^{(\max )}-\xi^{(\min )}}\right)^{2}} .
$$


The constant mixing ratios that bound the initial profile are

$$
\begin{array}{cc}
\chi^{(\min )}=0, & \chi^{(\max )}=1.0, \\
\xi^{(\min )}=0.1, & \xi^{(\max )}=0.9 .
\end{array}
$$

The mixing diagnostics work based on the classification of each element pair $\left(\chi_{k}, \xi_{k}\right)$ into region $\mathcal{A}, \mathcal{B}$ or $(\mathcal{A} \cup \mathcal{B})^{\prime}$. The mathematical descriptions of $\mathcal{A}$ and $\mathcal{B}$ are

$$
\begin{aligned}
\mathcal{A}= & \left\{(\chi, \xi) \mid \chi_{k} \in\left[\chi^{(\min )}, \chi^{(\max )}\right]\right. \\
& \text { and } \left.\mathcal{F}\left(\chi_{k}\right) \leq \xi_{k} \leq \psi\left(\chi_{k}\right)\right\} \\
\mathcal{B}= & \left\{(\chi, \xi) \mid\left(\chi_{k}, \xi_{k}\right) \in\left[\chi^{(\min )}, \chi^{(\max )}\right] \times\left[\xi^{(\min )}, \xi^{(\max )}\right]\right. \\
& \text { and } \left.\left(\chi_{k}, \xi_{k}\right) \notin \mathcal{A}\right\}
\end{aligned}
$$

where $\mathcal{F}$ is the straight line that connects $\left(\chi^{(\min )}, \xi^{(\max )}\right)$ and $\left(\chi^{(\max )}, \xi^{(\min )}\right)$.

The diagnostic for mixing that resembles 'real' mixing is defined as

$$
\ell_{\mathrm{r}} \equiv \frac{1}{A} \sum_{k} \begin{cases}d_{k} \Delta A_{k}, & \text { if }\left(\chi_{k}, \xi_{k}\right) \in \mathcal{A}, \\ 0 & \text { otherwise. }\end{cases}
$$

The diagnostic for mixing that is range-preserving is

$$
\ell_{\mathrm{u}} \equiv \frac{1}{A} \sum_{k} \begin{cases}d_{k} \Delta A_{k}, & \text { if }\left(\chi_{k}, \xi_{k}\right) \in \mathcal{B} \\ 0 & \text { otherwise. }\end{cases}
$$

Finally the diagnostic for overshooting is

$$
\ell_{\mathrm{o}} \equiv \frac{1}{A} \sum_{k} \begin{cases}d_{k} \Delta A_{k}, & \text { if }\left(\chi_{k}, \xi_{k}\right) \notin \mathcal{A} \cup \mathcal{B}, \\ 0 & \text { otherwise. }\end{cases}
$$

In all cases the summation is taken over all cells $k$.

For simplicity, and to prevent these calculations using very large file sizes, we chose to constrict our analysis to the five equidistantly spaced model levels at 4500, 4700, 4900, 5100 and $5300 \mathrm{~m}$ when calculating the mixing diagnostics and correlation plots for test $1-1$.

\section{Appendix: Perceived vertical velocity for test 1-3}

We are interested in writing a purely horizontal velocity field $\mathbf{u}$ in both a coordinate-following basis and a Cartesian basis. This procedure allows us to identify the source of the 'perceived' vertical velocities that may be caused by an underlying terrainfollowing vertical coordinate system with sloping coordinate surfaces. The basis-vector-following coordinate lines can be decomposed into a purely horizontal velocity and a purely vertical velocity. Mathematically, this takes the form

$$
\mathbf{g}_{s}=\left(\frac{\partial z}{\partial x}\right)_{s} \mathbf{g}_{z}+\mathbf{g}_{x}
$$

where $x$ is an arbitrary horizontal coordinate (such as $\lambda$ or $\varphi$ ), $z$ is the height coordinate, $s$ denotes the quantity that is constant along coordinate lines and $\mathbf{g}_{s}, \mathbf{g}_{z}$ and $\mathbf{g}_{x}$ denote basis vectors along surfaces of constant $s, z$ and $x$, respectively.

Given a velocity field in coordinate-following spherical coordinates (with basis vectors $\mathbf{g}_{\hat{\lambda}}$ and $\mathbf{g}_{\hat{\varphi}}$ ), we have

$$
\begin{aligned}
& \mathbf{g}_{\hat{\lambda}}=\frac{1}{a \cos \varphi}\left(\frac{\partial z}{\partial \lambda}\right)_{s} \mathbf{g}_{z}+\mathbf{g}_{\lambda}, \\
& \mathbf{g}_{\hat{\varphi}}=\frac{1}{a}\left(\frac{\partial z}{\partial \varphi}\right)_{s} \mathbf{g}_{z}+\mathbf{g}_{\varphi} .
\end{aligned}
$$

For test 1-3, we impose a purely horizontal velocity field $\mathbf{u}=u_{\lambda} \mathbf{g}_{\lambda}+u_{\varphi} \mathbf{g}_{\varphi}$ on the sphere (horizontal with respect to the 'main sea level'). Consequently, in coordinate-following spherical coordinates we have

$$
\begin{aligned}
\mathbf{u}= & u_{\lambda}\left[\mathbf{g}_{\hat{\lambda}}-\frac{1}{a \cos \varphi}\left(\frac{\partial z}{\partial \lambda}\right)_{s} \mathbf{g}_{z}\right] \\
& +u_{\varphi}\left[\mathbf{g}_{\hat{\varphi}}-\frac{1}{a}\left(\frac{\partial z}{\partial \varphi}\right)_{s} \mathbf{g}_{z}\right], \\
= & u_{\lambda} \mathbf{g}_{\hat{\lambda}}+u_{\varphi} \mathbf{g}_{\hat{\varphi}}+\left[-\frac{u_{\lambda}}{a \cos \varphi}\left(\frac{\partial z}{\partial \lambda}\right)_{s}-\frac{u_{\varphi}}{a}\left(\frac{\partial z}{\partial \varphi}\right)_{s}\right] \mathbf{g}_{z} .
\end{aligned}
$$

The basis vector for the last term in (D5) is $\mathbf{g}_{z}$. Therefore, we observe that in coordinate-following form we have introduced the additional 'perceived' vertical velocity:

$$
w=-\frac{u_{\lambda}}{a \cos \varphi}\left(\frac{\partial z}{\partial \lambda}\right)_{s}-\frac{u_{\varphi}}{a}\left(\frac{\partial z}{\partial \varphi}\right)_{s},
$$

where $u_{\lambda}$ and $u_{\varphi}$ are the zonal and meridional velocities with respect to the mean sea level. Here, they coincide with $u$ and $v$ shown in (42) and (43). The 'perceived' vertical velocity depends on how coordinate surfaces vary with height. The derivatives in (D6) are taken along the sloping coordinate surfaces (surfaces of constant generalized vertical coordinate $s$ ). Once $w$ is computed, the corresponding perceived vertical pressure velocity $\omega$ is given by (39).

To demonstrate how the perceived vertical velocity is computed, we present it in the height-based orography-following coordinate of Gal-Chen and Somerville (1975) (here denoted GC). The formulation for the perceived vertical velocity for the hybrid pressure-based $\eta$ coordinate (Simmons and Burridge, 1981), which is often used in hydrostatic dynamical cores, is also shown. If other vertical coordinates are used, the formulation for the vertical velocity needs to be newly derived according to the algorithm given here.

\section{D.1. Gal-Chen vertical coordinate}

The GC vertical coordinate $\bar{z} \in\left[0, z_{\text {top }}\right]$ maps to the range $z \in\left[z_{\mathrm{s}}(\lambda, \varphi), z_{\text {top }}\right]$. It is defined as

$$
\bar{z}=z_{\mathrm{top}}\left(\frac{z-z_{\mathrm{s}}(\lambda, \varphi)}{z_{\mathrm{top}}-z_{\mathrm{s}}(\lambda, \varphi)}\right)
$$

where $z_{\mathrm{s}}(\lambda, \varphi)$ is the surface elevation, for instance defined by (46), and $z_{\text {top }}$ is the height position of the model top. Coordinate surfaces in Cartesian space are defined via the inverse of (D7),

$$
z=z_{\mathrm{s}}(\lambda, \varphi)+\frac{\bar{z}}{z_{\mathrm{top}}}\left[z_{\mathrm{top}}-z_{\mathrm{s}}(\lambda, \varphi)\right]
$$

To compute the perceived vertical velocity, we differentiate (D8) along surfaces of constant $\bar{z}$, obtaining

$$
\begin{aligned}
\frac{\partial z}{\partial \lambda} & =\frac{\partial z_{\mathrm{s}}}{\partial \lambda}\left(1-\frac{\bar{z}}{z_{\mathrm{top}}}\right), \\
\frac{\partial z}{\partial \varphi} & =\frac{\partial z_{\mathrm{s}}}{\partial \varphi}\left(1-\frac{\bar{z}}{z_{\mathrm{top}}}\right) .
\end{aligned}
$$

The final step in this procedure requires one to compute the horizontal derivatives of $z_{\mathrm{s}}$ with respect to $\lambda$ and $\varphi$. Using the Schär mountain profile (46), the derivatives of the surface 
elevation are given as follows:

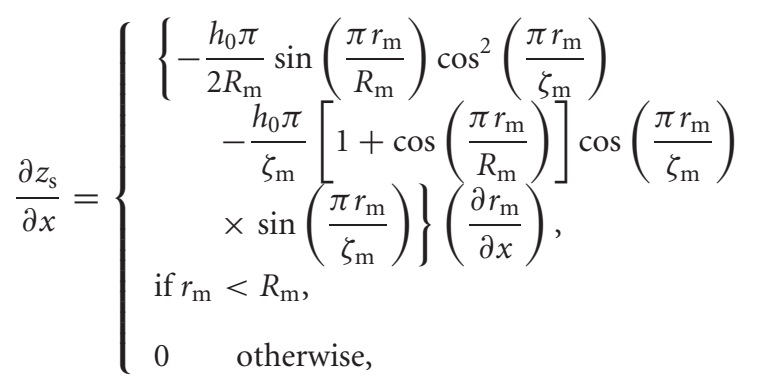

where $x \in\{\lambda, \varphi\}$ and

$$
\begin{aligned}
\frac{\partial r_{\mathrm{m}}}{\partial \lambda} & =\frac{\cos \varphi_{\mathrm{m}} \cos \varphi \sin \left(\lambda-\lambda_{\mathrm{m}}\right)}{\sqrt{1-\cos ^{2}\left[r_{\mathrm{m}}(\lambda, \varphi)\right]}}, \\
\frac{\partial r_{\mathrm{m}}}{\partial \varphi} & =\frac{-\sin \varphi_{\mathrm{m}} \cos \varphi+\cos \varphi_{\mathrm{m}} \sin \varphi \cos \left(\lambda-\lambda_{\mathrm{m}}\right)}{\sqrt{1-\cos ^{2}\left[r_{\mathrm{m}}(\lambda, \varphi)\right]}} .
\end{aligned}
$$

Note that when $r_{\mathrm{m}}(\lambda, \varphi)=0$ or $\pm \pi$, which will occur at $(\lambda, \varphi)=\left(\lambda_{\mathrm{m}}, \varphi_{\mathrm{m}}\right)$ or $\left(\lambda_{\mathrm{m}} \pm \pi,-\varphi_{\mathrm{m}}\right)$, we enforce $\partial r_{\mathrm{m}} / \partial \lambda=0$ and $\partial r_{\mathrm{m}} / \partial \varphi=0$. At each coordinate $(\lambda, \varphi)$, the set of equations (D6) - (D13) then leads to a unique perceived velocity associated with the terrain-following coordinate transform.

\section{D.2. Hybrid $\eta$ vertical coordinate}

Calculation of the perceived vertical velocity under hybrid $\eta$ coordinates requires the computation of the horizontal derivatives of $z$ with respect to $\lambda$ and $\varphi$. Under hybrid $\eta$ coordinates, we use the fact that $p=a(\eta) p_{0}+b(\eta) p_{\mathrm{s}}(\lambda, \varphi)$ (Simmons and Burridge, $1981)$. Combining this with the pressure equation for isothermal conditions with temperature $T_{0}$ and reference surface pressure $p_{0}$, we obtain

$$
z=-\frac{R_{\mathrm{d}} T_{0}}{g} \ln \left[a(\eta)+b(\eta) \frac{p_{\mathrm{s}}(\lambda, \varphi)}{p_{0}}\right] .
$$

Consequently,

$$
\begin{aligned}
& \left(\frac{\partial z}{\partial \lambda}\right)_{\eta}=-\frac{R_{\mathrm{d}} T_{0}}{g}\left[a(\eta)+b(\eta) \frac{p_{\mathrm{s}}(\lambda, \varphi)}{p_{0}}\right]^{-1} \frac{b(\eta)}{p_{0}} \frac{\partial p_{\mathrm{s}}}{\partial \lambda}, \\
& \left(\frac{\partial z}{\partial \varphi}\right)_{\eta}=-\frac{R_{\mathrm{d}} T_{0}}{g}\left[a(\eta)+b(\eta) \frac{p_{\mathrm{s}}(\lambda, \varphi)}{p_{0}}\right]^{-1} \frac{b(\eta)}{p_{0}} \frac{\partial p_{\mathrm{s}}}{\partial \varphi} .
\end{aligned}
$$

Equivalently,

$$
\begin{aligned}
& \left(\frac{\partial z}{\partial \lambda}\right)_{\eta}=-\frac{R_{\mathrm{d}} T_{0}}{g p} b(\eta) \frac{\partial p_{\mathrm{s}}}{\partial \lambda}, \\
& \left(\frac{\partial z}{\partial \varphi}\right)_{\eta}=-\frac{R_{\mathrm{d}} T_{0}}{g p} b(\eta) \frac{\partial p_{\mathrm{s}}}{\partial \varphi} .
\end{aligned}
$$

Since the surface profile is given in terms of height $z$, we need to use

$$
\frac{\partial p_{s}}{\partial x}=-\frac{g p_{0}}{R_{\mathrm{d}} T_{0}} \exp \left(\frac{-g z_{\mathrm{s}}}{R_{\mathrm{d}} T_{0}}\right) \frac{\partial z_{\mathrm{s}}}{\partial x},
$$

where $x$ denotes a placeholder $(x \in\{\lambda, \varphi\})$ and $\partial z_{s} / \partial x$ is again given by (D11)-(D13). Since pressure is constant with time, the pressure velocity is also related to the vertical velocity via equation (39).

In practice, the following steps can be used to apply the perceived vertical velocity under a hybrid $\eta$ vertical coordinate:
(1) Compute the surface height derivatives from (D11)-(D13).

(2) Compute the surface pressure derivatives from (D19).

(3) For each coordinate line (vertical edge), compute (D17)-(D18).

(4) Compute the perceived vertical velocity $w$ from (D6).

(5) Compute the perceived pressure velocity $\omega$ from (39).

\section{Acknowledgements}

We thank all the participants of the 2012 Dynamical Core Model Intercomparison Project (DCMIP) Workshop. We also thank Robert Walko for outlining a strategy that led to the development of test $1-3$.

This research was supported by the Department of Energy (DoE) Office of Science awards DE-SC0003990 and DESC0006684.

\section{Supporting information}

The following supporting information is available as part of the online article:

Table S1. FORTRAN initialization routines for our test cases. The initialization routines as three subroutines in a FORTRAN .f90 file. The routines must be called for each grid point, therefore the latitude, longitude and height or pressure are inputted, and the output contains the velocities, tracers and other dynamic variables (for example surface pressure).

\section{References}

Colella P, Woodward PR. 1984. The Piecewise Parabolic Method (PPM) for gas-dynamical simulations. J. Comput. Phys. 54: 174-201.

Gal-Chen T, Somerville RCJ. 1975. On the use of a coordinate transformation for the solution of the Navier-Stokes equations. J. Comput. Phys 17: 209-228.

Hubbard ME. 2002. Adaptive mesh refinement for three-dimensional off-line tracer advection over the sphere. Int. J. Numer. Methods Fluids 40: 369-377.

Kent J, Jablonowski C, Whitehead JP, Rood RB. 2012a. Assessing tracer transport algorithms and the impact of vertical resolution in a finite-volume dynamical core. Mon. Weather Rev. 140: 1620-1638.

Kent J, Jablonowski C, Whitehead JP, Rood RB. 2012b. Downscale cascades in tracer transport test cases: an intercomparison of the dynamical cores in the Community Atmosphere Model CAM5. Geosci. Model Dev. 5: 1517-1530.

Lamarque J-F, Kinnison DE, Hess PG, Vitt FM. 2008. Simulated lower stratospheric trends between 1970 and 2005: Identifying the role of climate and composition changes. J. Geophys. Res. 113: D12301. DOI:10.1029/2007JD009277.

Laprise R, Girard C. 1990. A spectral general circulation model using a piecewise-constant finite-element representation on a hybrid vertical coordinate system. J. Climate 3: 32-52.

Lauritzen PH, Thuburn J. 2012. Evaluating advection/transport schemes using interrelated tracers, scatter plots and numerical mixing diagnostics. Q. J. R. Meteorol. Soc. 138: 906-918.

Lauritzen PH, Skamarock WC, Prather MJ, Taylor MA. 2012. A standard test case suite for two-dimensional linear transport on the sphere. Geosci. Model Dev. 5: 887-901.

Lin SJ. 2004. A ‘vertically Lagrangian' finite-volume dynamical core for global models. Mon. Weather Rev. 132: 2293-2307.

Lin SJ, Rood RB. 1996. Multidimensional flux-form semi-Lagrangian transport schemes. Mon. Weather Rev. 124: 2046-2070.

Nair RD, Jablonowski C. 2008. Moving vortices on the sphere: a test case for horizontal advection problems. Mon. Weather Rev. 136: 699-711.

Nair RD, Lauritzen PH. 2010. A class of deformational flow test cases for linear transport problems on the sphere. J. Comput. Phys. 229: 8868-8887.

Nair RD, Machenhauer B. 2002. The mass-conservative cell-integrated semiLagrangian advection scheme on the sphere. Mon. Weather Rev. 130: 649-667.

Nair RD, Thomas SJ, Loft RD. 2005. A discontinuous Galerkin transport scheme on the cubed sphere. Mon. Weather Rev. 133: 814-828.

Neale RB, Chen C-C, Gettelman A, Lauritzen PH, Park S, Williamson DL, Conley AJ, Garcia R, Kinnison D, Lamarque J-F, Marsh D, Mills M, Smith AK, Tilmes S, Vitt F, Cameron-Smith P, Collins WD, Iacono MJ, Rasch PJ, Taylor MA. 2010. 'Description of the NCAR Community Atmosphere Model (CAM 5.0)', NCAR Tech. Note NCAR/TN-486+STR. NCAR: Boulder, CO, 268.

Ovtchinnikov M, Easter RC. 2009. Nonlinear advection algorithms applied to interrelated tracers: Errors and implications for modeling aerosol-cloud interactions. Mon. Weather Rev. 137: 632-644. 
Phillips NA. 1957. A coordinate system having some special advantages for numerical forecasting, J. Meteorol. 14: 184-185.

Plumb RA, Ko MKW. 1992. Interrelationships between mixing ratios of long-lived stratospheric constituents. J. Geophys. Res. 97: 10 145-10 156.

Plumb RA, Waugh DW, Chipperfield MP. 2000. The effects of mixing on tracer relationships in the polar vortices. J. Geophys. Res. 105: 10 047-10 062.

Prather MJ, Zhu X, Strahan SE, Steenrod SD, Rodriguez JM. 2008. Quantifying errors in trace species transport modeling. Proc. Natl Acad. Sci. 150: 19617-19621.

Rancic M, Purser RJ, Mesinger F. 1996. A global shallow-water model using an expanded spherical cube: Gnomonic verse conformal coordinates. Q. J. $R$. Meteorol. Soc. 122: 959-982.

Rasch PJ, Coleman DB, Mahowald N, Williamson DL, Lin SJ, Boville BA, Hess P. 2006. Characteristics of atmospheric transport using three numerical formulations for atmospheric dynamics in a single GCM framework. J. Climate 19: 2243-2266.

Rood RB. 1987. Numerical advection algorithms and their role in atmospheric transport and chemistry models. Rev. Geophys. 25: 71-100.

Schär C, Leuenberger D, Fuhrer O, Luthi D, Girard C. 2002. A new terrainfollowing vertical coordinate formulation for atmospheric prediction models. Mon. Weather Rev. 130: 2459-2480.

Simmons AJ, Burridge DM. 1981. An energy and angular-momentum conserving vertical finite-difference scheme and hybrid vertical coordinates. Mon. Weather Rev. 109: 758-766.

Staniforth A, Thuburn J. 2012. Horizontal grids for global weather and climate prediction models: A review. Q. J. R. Meteorol. Soc. 138: 1-26.
Thuburn J, Mclntyre M. 1997. Numerical advection schemes, cross- isentropic random walks, and correlations between chemical species. J. Geophys. Res. 102: 6775-6797.

Ullrich PA, Jablonowski C. 2012a. MCore: A nonhydrostatic atmospheric dynamical core utilizing high-order finite-volume methods. J. Comp. Phys. 231: $5078-5108$.

Ullrich PA, Jablonowski C. 2012b. Operator-split Runge-Kutta-Rosenbrock (RKR) methods for nonhydrostatic atmospheric models. Mon. Weather Rev. 140: $1257-1284$.

Ullrich PA, Jablonowski C, van Leer B. 2010. High-order finite-volume models for the shallow-water equations on the sphere. J. Comp. Phys. 229: 6104-6134.

Whitehead JP, Jablonowski C, Kent J, Rood RB. 2013. Potential vorticity: Measuring consistency between GCM dynamical cores and tracer advection schemes. Q. J. R. Meteorol. Soc. Submitted.

Williamson DL, Drake JB, Hack JJ, Jakob R, Swarztrauber PN. 1992. A standard test set for numerical approximations to the shallow water equations in spherical geometry. J. Comput. Phys. 102: 211-224.

Zerroukat M, Allen T. 2012. A three-dimensional monotone and conservative semi-Lagrangian scheme (SLICE-3D) for transport problems. Q. J. R. Meteorol. Soc. 138: 1640-1651.

Zerroukat M, Wood N, Staniforth A. 2002. SLICE: A semi-Lagrangian inherently conserving and efficient scheme for transport problems. $Q$. J. R. Meteorol. Soc. 128: 2801-2820.

Zubov VA, Rozanov EV, Schlesinger ME. 1999. Hybrid scheme for threedimensional advection transport. Mon. Weather Rev. 127: 1335-1346. 